\title{
ERGODIC THEOREMS FOR COSET SPACES
}

\author{
MICHAEL BJÖRKLUND AND ALEXANDER FISH
}

\begin{abstract}
We study in this paper the validity of the mean ergodic theorem along left Følner sequences in a countable amenable group $G$. Although the weak ergodic theorem always holds along any left Følner sequence in $G$, we provide examples where the mean ergodic theorem fails in quite dramatic ways. On the other hand, if $G$ does not admit any ICC quotients, e.g. if $G$ is virtually nilpotent, then we prove that the mean ergodic theorem does indeed hold along any left Følner sequence.

In the case when a unitary representation of a countable amenable group is induced from a unitary representation of a "sufficiently thin" subgroup, we prove that the mean ergodic theorem holds along any left Følner sequence for this representation.

Furthermore, we show that every countable (infinite) amenable group $L$ embeds into a countable group $G$ which admits a unitary representation with the property that for any left Følner sequence $\left(F_{n}\right)$ in $L$, there exists a sequence $\left(s_{n}\right)$ in $G$ such that the mean (but not the weak) ergodic theorem fails for this representation along the sequence $\left(F_{n} s_{n}\right)$.

Finally, we provide examples of countable (not necessarily amenable) groups $G$ with proper, infinite-index subgroups $H$, so that the pointwise ergodic theorem holds for averages along any strictly increasing and nested sequence of finite subsets of the coset $G / H$.
\end{abstract}

\section{INTRODUCTION}

1.1. General comments. We recall that the celebrated strong (mean) ergodic theorem of J. von Neumann asserts that whenever $U$ is a unitary operator on a Hilbert space $\mathscr{H}$, then all of the limits

$$
\lim _{n} \frac{1}{n} \sum_{k=0}^{n-1} U^{k} v=P_{U} v
$$

exist in the norm topology on $\mathscr{H}$ for every $v \in \mathscr{H}$, where $P_{U}$ denotes the orthogonal projection onto the closed linear subspace $\mathscr{H}^{U} \subset \mathscr{H}$ spanned by all $U$-invariant vectors. More generally, we can consider a unitary representation $(\mathscr{H}, \pi)$ of a countable group $G$ and ask for conditions on sequences $\left(F_{n}\right)$ of finite subsets of $G$ which are strong enough to ensure that the limits

$$
\lim _{n} \frac{1}{\left|F_{n}\right|} \sum_{s \in F_{n}} \pi(s) v=P_{G} v
$$

exist in the norm (or weak) topology on $\mathscr{H}$ for every $v \in \mathscr{H}$, where $P_{G}$ denotes the orthogonal projection onto the closed linear subspace $\mathscr{H}^{G} \subset \mathscr{H}$ spanned by all $\pi(G)$-invariant vectors. We shall say that such sequences are strong for $(\mathscr{H}, \pi)$, or weak if the limits are only guaranteed to exist in the weak topology on $\mathscr{H}$. Clearly, every strong sequence is a weak sequence for any unitary representation.

As was pointed out by W. F. Eberlein in [10] (based on some fundamental observations by F. Riesz in [22]), if $G$ is a countable amenable group, then every right Følner sequence in $G$ is a

2010 Mathematics Subject Classification. Primary: 37A30; Secondary: 22D10, 43A07.

Key words and phrases. Ergodic theorems, Unitary representations, Amenability. 
strong sequence for every unitary representation of $G$. We recall that a sequence $\left(F_{n}\right)$ of finite subsets of $G$ is a right Følner sequence if

$$
\lim _{n} \frac{\left|F_{n} \triangle F_{n} t\right|}{\left|F_{n}\right|}=0, \quad \text { for all } t \in G
$$

and it is a left Følner sequence if

$$
\lim _{n} \frac{\left|F_{n} \Delta t F_{n}\right|}{\left|F_{n}\right|}=0, \quad \text { for all } t \in G
$$

and we say that a countable group is amenable if it admits a right Følner sequence. Since the inverse of any right Følner sequence is a left Følner sequence, we could just as well have required in the definition of an amenable group that it should admit a left Følner sequence.

The class of amenable groups is quite large and contains all solvable (hence all abelian) groups, all locally finite groups and all finitely generated groups of subexponential growth. However, free groups on two or more generators and countably infinite groups with property (T) are well-known examples of non-amenable groups.

If $G$ is abelian (or more generally, a finite extension of an abelian group), then every left Følner sequence in $G$ is also a right Følner sequence. However, this property is very special for this class of groups, and we note that if $G$ has at least one element with an infinite conjugation class, then there is a left Følner sequence which is very far from being a right Følner sequence; indeed, let $\left(F_{n}\right)$ be any left Følner sequence in such a group and suppose that $t$ is an element in $G$ with an infinite conjugation class. Then we can find a sequence $\left(s_{n}\right)$ in $G$ such that the sets $F_{n}$ and $F_{n} s_{n} t s_{n}^{-1}$ are disjoint for every index $n$, and thus the left Følner sequence $\left(F_{n} s_{n}\right)$ satisfies

$$
\frac{\left|F_{n} s_{n} \triangle F_{n} s_{n} t\right|}{\left|F_{n}\right|}=\frac{\left|F_{n} \triangle F_{n} s_{n} t s_{n}^{-1}\right|}{\left|F_{n}\right|}=2 \text { for all } n,
$$

which shows that $\left(F_{n} s_{n}\right)$ is not a right Følner sequence.

It is not hard to prove (and we shall give the proof in Section 3) that left Følner sequences in any countable amenable group $G$ are weak sequences for any unitary representation of $G$. One of the main aims of this paper is to investigate to which extent left Følner sequences in countable amenable groups are also strong sequences (at least for large classes of unitary representations).

1.2. Left amenable triples. We shall study a slightly more general setting than the one described in the previous subsection. We begin by introducing some of the central concepts needed to state and explain the main results of this paper.

Definition 1.1 (Strong and weak sequences). Let $G$ be a countable group and let $H$ and $L$ be subgroups of $G$. We shall say that a sequence $\left(F_{n}\right)$ of finite subsets of $G / H$ is a strong (weak) sequence for the triple $(G, H, L)$ if the limits

$$
\lim _{n} \frac{1}{\left|F_{n}\right|} \sum_{s \in F_{n}} \pi(s) v=P_{L} v
$$

exist in the norm (weak) topology on $\mathscr{H}$ for all $v \in \mathscr{H}^{H}$ (the closed linear subspace of $\mathscr{H}$ spanned by $\pi(H)$-invariant vectors).

We refer the reader to Section 2 for a list of examples of triples of countable groups, some of which have been extensively studied in the literature. 
Given a triple $(G, H, L)$ as in the definition above, we note that $G$, and hence the subgroup $L$, always admits a left action on the coset space $G / H$, and with respect to this action, the following extension of the notion of a left Følner sequence seems natural.

Definition 1.2 (Left Følner sequence). We say that a sequence $\left(F_{n}\right)$ of finite subsets of $G / H$ is a left Følner sequence for $(G, H, L)$ if

$$
\lim _{n} \frac{\left|F_{n} \triangle s F_{n}\right|}{\left|F_{n}\right|}=0, \quad \text { for all } s \text { in } L
$$

and we say that the triple $(G, H, L)$ is left amenable if it admits a left Følner sequence.

Remark 1.1. We stress that the notion of left amenable triples is not a new, although in the literature it is more commonplace to write that $L$ acts amenably on $G / H$. Also, in the special case when the triple $(G, H, G)$ is left amenable, one often says that $H$ is co-amenable in $G$. It is not hard to show that if $G$ is an amenable group, then $(G, H, L)$ is left amenable for any pair of subgroups $H$ and $L$ of $G$.

The proof of the following theorem is not hard, but for completeness we have decided to give the short proof in Section 3 .

Theorem 1.1 (Left Weak Ergodic Theorem). Every left Følner sequence for a left amenable triple is a weak sequence for the same triple.

We recall that if $(\mathscr{H}, \pi)$ is a unitary representation of a countable group $G$, then we can decompose $\mathscr{H}$ into an orthogonal direct sum of two sub-representations $\mathscr{H}_{\text {fin }}$ and $\mathscr{H}_{\mathrm{wm}}$, where $\mathscr{H}_{\text {fin }}$ denotes the closed linear span of all of those vectors in $\mathscr{H}$ whose cyclic linear spans are finite-dimensional, and thus its orthogonal complement $\mathscr{H}_{\mathrm{wm}}$ in $\mathscr{H}$ has no finite-dimensional sub-representations. We say that the unitary representation $\left(\mathscr{H}_{\text {fin }},\left.\pi\right|_{\mathcal{H}_{\text {fin }}}\right)$ has pure point spectrum and we say that $\left(\mathscr{H}_{\mathrm{wm}},\left.\pi\right|_{\mathscr{H}_{\mathrm{wm}}}\right)$ is weakly mixing.

Since the weak topology and the norm topology coincide on finite-dimensional Hilbert spaces, we have the following corollary of Theorem 1.1 .

Corollary 1.1. If $G$ is a countable amenable group, then every left Følner sequence in $G$ is a strong sequence for any unitary representation with pure point spectrum.

We note that if we want to show that a given sequence $\left(F_{n}\right)$ of finite subsets of a countable group $G$ is a strong sequence for any unitary representation, then it suffices to show this for irreducible unitary representations (see e.g. Proposition 2.3.2. in [28]). In particular, by the previous corollary, we conclude that left Følner sequences are always strong for the class of countable groups (so called Moore groups) all of whose irreducible unitary representations are finite-dimensional.

Corollary 1.2 (von Neumann's Ergodic Theorem). If $G$ is a countable Moore group, then every left Folner sequence in $G$ is a strong sequence for any unitary representation.

Every countable abelian group is a Moore group, and conversely, any countable Moore group is a finite extension of a countable abelian group, which was proved by $\mathrm{E}$. Thoma in [25].

1.3. Right amenable triples. We note here that in some cases of interest, it is also possible to define a natural notion of a right Følner sequence for a triple. In order to explain this notion, let $N_{G}(H)$ denote the normalizer of $H$ in $G$, and observe that $N_{G}(H)$ admits an action on the coset space $G / H$ on the right via the map

$$
c_{H}(s) x H=x s^{-1} H \quad \text { for } s \in N_{G}(H) .
$$


This $N_{G}(H)$-action commutes with the left action of $G$ on the coset space $G / H$ and factors through the quotient $H \backslash N_{G}(H)$. We can now extend the notion of a right Følner sequence to the setting of triples as follows.

Definition 1.3 (Right Følner sequence). Suppose that the inclusions $H<L<N_{G}(H)$ hold. We say that a sequence $\left(F_{n}\right)$ of finite subsets of $L / H$ is a right Følner sequence for $(G, H, L)$ if

$$
\lim _{n} \frac{\left|F_{n} \triangle c_{H}(s) F_{n}\right|}{\left|F_{n}\right|}=0, \text { for all } s \text { in } L .
$$

We say that the triple $(G, H, L)$ is right amenable if it admits a right Følner sequence $\left(F_{n}\right)$ such that $\left|F_{n}\right| \rightarrow \infty$. In the special case when $L=G$ and $H$ is the trivial subgroup, this notion coincides with the definition of a right Følner sequence in $G$ given above.

Remark 1.2. If the quotient group $L / H$ is an infinite amenable group and $\left(K_{n}\right)$ is a right $F \varnothing l-$ ner sequence in $L / H$, then $\left(c_{H}\left(K_{n}^{-1}\right) H\right)$ is a right Følner sequence for $(G, H, L)$. We stress that the notion of a right Følner sequence in this generality is much more restrictive than the corresponding notion of a left Følner sequence. For instance, since right Følner sequences are assumed to be contained in the coset space $L / H$, the notion of right amenability (existence of a right Følner sequence whose sizes tend to infinite) only makes sense if $L / H$ itself is infinite. In particular, right amenability is essentially a void concept if $H$ is self-normalized in $G$, that is to say, if $N_{G}(H)=H$.

In Section 4 we shall adapt a classical argument of F. Riesz to the setting of triples and establish the following strong ergodic theorem.

Theorem 1.2 (Right Strong Ergodic Theorem). Every right Følner sequence for a right amenable triple is a strong sequence for the same triple.

An unfortunate feature with right Følner sequences for a right amenable triple $(G, H, L)$ is that one cannot really hope to gain more than $L$-invariance upon averaging unitary representations with $\pi(H)$-invariant vectors. In particular, if the normalizer of $H$ in $G$ is small, then this may not be very useful.

1.4. A transfer principle. We shall now return to our discussion concerning the validity of the mean ergodic theorem along left Følner sequences.

Let $(G, H, L)$ be a left amenable triple and let $(\mathscr{H}, \pi)$ be a unitary representation of $G$ with non-zero $\pi(H)$-invariant vectors. We say that the Left Strong Ergodic Theorem holds for $(\mathcal{H}, \pi)$ if every left Følner sequence for $(G, H, L)$ is strong for $(\mathcal{H}, \pi)$, that is to say, if the limits

$$
\lim _{n} \frac{1}{\left|F_{n}\right|} \sum_{s \in F_{n}} \pi(s) v=P_{L} v
$$

exists in the norm topology for every $v \in \mathscr{P}^{H}$ and for every left Følner sequence for $(G, H, L)$. If this is the case for every unitary representation of $G$, then we simply say that the Strong Left Ergodic Theorem holds for the triple $(G, H, L)$, and in the special case when $G=L$ and $H$ is the trivial subgroup, we say that the Strong Left Ergodic Theorem holds for $G$.

Our first main contribution to this paper is the observation that the question whether the Left Strong Ergodic Theorem holds for a given countable amenable group can be equivalently phrased in a way which does not use left Følner sequences (and hence not amenability). The key notion behind this reformulation is that of a firm strong sequence, which we define as follows. 
Definition 1.4 (Firm strong sequence). Let $(\mathscr{H}, \pi)$ be a unitary representation of a countable (not necessarily amenable) group $G$. A sequence $\left(K_{m}\right)$ of finite subsets of $G$ is called a firm strong sequence with respect to $(\mathscr{H}, \pi)$ if for every choice of a sequence $\left(t_{m}\right)$ in $G$, we have

$$
\lim _{n} \frac{1}{\left|K_{m}\right|} \sum_{t \in K_{m}} \pi\left(t t_{m}\right) v=P_{G} v
$$

for all $v \in \mathscr{H}$ in the norm topology on $\mathscr{H}$. If $\left(K_{m}\right)$ is a firm strong sequence for every unitary representation of $G$, then we shall simply say that $\left(K_{m}\right)$ is a strong firm sequence in $G$.

We note that if $G$ is a countable abelian group, then every strong sequence in $G$ is firm, but as we shall see below, many countable (amenable and non-amenable) groups do not admit firm sequences. In fact, we have the following equivalence result.

Proposition 1.1 (Transfer principle). The Strong Left Ergodic Theorem holds for a countable amenable group if and only if the group admits a firm strong sequence.

In particular, this theorem allows us to "localize" the question whether the Left Strong Ergodic Theorem holds along any left Følner sequence to whether it holds along all right translates of a fixed sequence of finite subsets of the group (not necessarily a left Følner sequence).

1.5. The Left Strong Ergodic Theorem for countable nilpotent groups. We recall that a countable group $G$ is ICC (Infinite Conjugation Classes) if every non-trivial element in $G$ has an infinite conjugation class. For instance, free groups on two or more generators are ICC, so are lattices in connected simple real Lie groups (a direct consequence of the Borel Density Theorem, see e.g. Proposition 2 in [4]) and many countable non-nilpotent solvable groups.

On the other hand, countable nilpotent groups have non-trivial centers and are thus never ICC. More generally, countable groups which contain finite-index nilpotent subgroups (so called virtually nilpotent groups) are never ICC.

We can now state our first main result in this paper as follows.

Theorem 1.3. If $G$ is a countable amenable group without ICC quotients, then every right Følner sequence in $G$ is a firm strong sequence. In particular, the Strong Left Ergodic Theorem holds for every countable virtually nilpotent group.

Remark 1.3. This result might seem a bit suspicious since there is absolutely no reason to expect that right translates of any right Følner sequence in $G$ should exhibit any asymptotic invariance whatsoever (either on the left or on the right hand side). We stress that the existence of finite conjugation classes in every quotient group of $G$ is the mechanism which will drive the argument.

The arguments in the proof of Theorem 1.3 will also yield the following corollary, and will be outlined in Subsection 6.1.

Corollary 1.3 (Reduction to ICC groups). Let $G$ be a countable amenable group for which the Left Strong Ergodic Theorem fails. Then G admits a quotient group which is ICC and for which the Left Strong Ergodic Theorem fails.

We shall later provide an example of a countable (infinitely generated) ICC group for which the Left Strong Ergodic Theorem fails in a very dramatic way. On the other hand, we have not been able to construct a single example of a countable amenable ICC group for which the Left Strong Ergodic Theorem holds for all unitary representations. Therefore it seems natural to ask the following question.

Question. Does the Left Strong Ergodic Theorem fail for every countable amenable ICC group? 
There are at least three reasons why this is an intricate question. The first reason is that we do not know at the moment whether there are countable (amenable) groups for which every unitary representation is mixing. Recall that a unitary representation $(\mathscr{H}, \pi)$ of a countable group $G$ is mixing if for every $v \in \mathscr{H}$ and $\varepsilon>0$, there exists a finite $\operatorname{set} F \subset G$ such that

$$
\sup _{s \in G \backslash F}|\langle v, \pi(s) v\rangle|<\varepsilon \text {. }
$$

One can readily check that every sequence of finite sets in $G$ whose sizes tend to infinity is a strong sequence for any mixing unitary representation of $G$, and thus the Left Strong Ergodic Theorem for these representations is immediate. The question whether countable (amenable) groups, all of whose unitary representations are mixing, exist was first raised by K. Schmidt in the paper [24], where it is proved that such groups must be finitely generated and can never admit proper infinite subgroups. In particular, possibly modulo a finite normal subgroup, such a group is ICC, but the Left Strong Ergodic Theorem would nevertheless hold for such a group.

The second reason why the question above is intricate has to do with the fact that there are many non-amenable ICC groups which admit firm strong sequences as the following proposition shows.

Proposition 1.2 (Gorodnik-Nevo, Theorem 1.7 in [13]). If $\tilde{G}$ is a non-compact and connected simple Lie group with trivial center and real rank at least two, e.g. $\mathrm{PSL}_{n}(\mathbb{R})$ for $n \geq 3$, then every lattice in $\tilde{G}$ is ICC and admits a firm strong sequence.

We stress that this is not how Theorem 1.7 in [13] is stated and for the reader's convenience we sketch in the appendix of this paper the derivation of the formulation above from that of Theorem 1.7 in [13]. The proof that lattices in non-compact and connected simple Lie groups with trivial centers are ICC (for this, the real rank assumption is unnecessary) utilizes the Borel Density Theorem and can be found in the paper [4] (Proposition 2).

1.6. A strong ergodic theorem for induced representations. The third reason why the question in the previous subsection is intricate stems from our very poor understanding of $i r$ reducible unitary representations of a general countable (discrete) group, which is not a finite extension of an abelian group.

In fact, for very large classes of countable groups, the only arsenal of unitary representations (irreducible or not) which we have at our disposal are the ones which are induced from various subgroups, whose unitary representation theory we know better.

We recall that if $G$ is a countable group and $L$ is a proper subgroup of $G$, then a unitary representation $(\mathscr{H}, \pi)$ of $G$ is induced from $L$, if there exists a unitary representation $\left(\mathscr{H}, \pi_{o}\right)$ of $L$ such that $(\mathscr{H}, \pi)$ is isomorphic to the right regular representation on the (pre-)Hilbert space

$$
\operatorname{Ind}_{L}^{G} \pi_{o}=\left\{f: G \rightarrow \mathscr{H}_{o}:\|f\|<\infty \text { and } f(l g)=\pi_{o}(l)^{-1} f(g), \quad \text { for all } l \in L \text { and } g \in G\right\} .
$$

Here, $\|\cdot\|$ denotes the norm induced from the following inner product

$$
\left\langle f, f^{\prime}\right\rangle=\sum_{x \in L \backslash G}\left\langle f(x), f^{\prime}(x)\right\rangle_{o},
$$

where $\langle\cdot, \cdot\rangle_{o}$ is the inner product on $\mathscr{H}_{o}$. In particular, we note that $\operatorname{Ind}_{L}^{G} \mathrm{id}_{L}$ is isomorphic to the left regular representation of $G$ on $\ell^{2}(G / L)$.

A classical and very useful result of G. Mackey (see e.g. Theorem 6 in [17]) asserts that if $L$ is a subgroup of a countable group $G$ such that every non-identity coset in $G / L$ has an infinite 
$L$-orbit, then for any finite-dimensional irreducible unitary representation $\left(\mathscr{H}_{o}, \pi_{o}\right)$ of $L$, the induced representation $\operatorname{Ind}_{L}^{G} \pi_{o}$ is irreducible. In particular, if $L$ is such a subgroup, then the left regular representation of $G$ on $\ell^{2}(G / L)$ is irreducible.

Our main result in this paper pertaining to induced representations, shows in particular that if $G$ is a countable amenable group and $L$ is a "sufficiently thin" subgroup in $G$, then induced representations from $L$ can never constitute counterexamples to the Left Strong Ergodic Theorem for the group $G$.

Proposition 1.3 (Ergodic theorem for induced representations). Let $G$ be a countable group and let $\left(F_{n}\right)$ be a sequence of finite subsets of $G$. Suppose that $L$ is a subgroup of $G$ which satisfies

$$
\lim _{n} \sup _{x, y \in G / L} \frac{\left|F_{n} \cap y L x^{-1}\right|}{\left|F_{n}\right|}=0 .
$$

If $\left(\mathscr{H}_{o}, \pi_{o}\right)$ is a unitary representation of $L$, then $\left(F_{n}\right)$ is a firm strong sequence with respect to the unitary induction $\operatorname{Ind}_{L}^{G} \pi_{o}$.

Remark 1.4. We stress that we do not assume that the Left Strong Ergodic Theorem holds for the unitary representation $\left(\mathcal{H}_{o}, \pi_{o}\right)$ of the subgroup $L$.

In Section $[7$ we shall prove this result and discuss the thinness criterion (1.5) for subgroups of semidirect products.

We stress that Proposition 1.3 does not imply that induced representations are never counterexamples to the Left Strong Ergodic Theorem. In fact, we shall in connection with Theorem 1.5 below introduce the notion of a conjugation-thick subgroup whose very definition is a strong violation of condition (1.5). We shall provide an example of a countable (amenable) group $G$ with a proper conjugation-thick subgroup $L$ and show that the Left Strong Ergodic Theorem fails in a very dramatic way for the left regular representation of $G$ on $\ell^{2}(G / L)$, which is the induction of the identity representation on $L$ to $G$.

1.7. Non-property (T) groups and flabby pairs. We shall now begin to discuss various failures of the Left Strong Ergodic Theorem in a more systematic fashion. For this, we need the notions of flabby pairs and flabby groups, where the latter can be seen as a very strong obstruction to the existence of a firm strong sequence in the group.

Definition 1.5 (Flabby pair). Let $G$ be a countable (not necessarily amenable) group and let $L$ be a subgroup of $G$. We say that the pair $(G, L)$ is flabby if there exists a unitary representation $(\mathcal{H}, \pi)$ of $G$ with no non-zero $\pi(L)$-invariant vectors with the property that for any sequence $\left(F_{n}\right)$ of finite subsets of $L$, whose sizes tend to infinity, there exists a sequence $\left(s_{n}\right)$ in $G$ such that

$$
\lim _{n}\left\|\frac{1}{\left|F_{n}\right|} \sum_{s \in F_{n}} \pi\left(s s_{n}\right) v\right\|=1
$$

for some unit vector $v$ in $\mathscr{H}$, and we say that $G$ is flabby if the pair $(G, G)$ is flabby.

We shall define and discuss Kazhdan's property (T) in Subsection 8 , where the following embedding theorem is proved.

Theorem 1.4 (Embedding into flabby pairs). Every countable group L without Kazhdan's property (T), for instance, every countable infinite amenable group, embeds into a countable group $G$ such that $(G, L)$ is a flabby pair. 
In the case when $L$ is a countable (infinite) amenable group, then $(G,\{e\}, L)$ is always a left amenable triple and $\left(F_{n} s_{n}\right)$ is a left Følner sequence for this triple whenever $\left(F_{n}\right)$ is a left Følner sequence in $L$ and $\left(s_{n}\right)$ is any sequence in $G$.

In particular, by the Left Weak Ergodic Theorem, $\left(F_{n} s_{n}\right)$ is a weak sequence for $(G,\{e\}, L)$. However, as Theorem 1.4 demonstrates, it is always possible to choose $\left(s_{n}\right)$ so that $\left(F_{n} s_{n}\right)$ is not a strong sequence for $(G,\{e\}, L)$, so the Left Strong Ergodic Theorem fails for this triple.

1.8. Explicit examples where the Left Strong Ergodic Theorem fails. Unfortunately, the construction of the embedding in Theorem 1.4 is far from explicit, and thus does not yield any insight to the question whether the Left Strong Ergodic Theorem can fail for a countable amenable group (and not just for a left amenable triple). As it turns out, the following notion of a contracting triple will provide a very convenient and concrete framework for discussing such failures.

Definition 1.6 (Contracting triples). Let $G$ be a countable group and let $H$ and $L$ be subgroups of $G$. The triple $(G, H, L)$ is called contracting if $H<L$ and for every finite subset $F \subset L$, there exists $t \in G$ such that $t F t^{-1} \subset H$.

In the case when $G=L$, we say that $H$ is a conjugation-thick subgroup.

We shall discuss various examples of contracting triples and conjugation-thick subgroups in Section 9. At this point of the exposition we shall simply state and prove the following key result about these triples.

Theorem 1.5 (Failure for contracting triples). If $(G, H, L)$ is a contracting triple such that $H$ has infinite index in $L$, then the pair $(G, L)$ is flabby. In particular, the Left Strong Ergodic Theorem fails for the triple $(G,\{e\}, L)$.

Indeed, suppose that $(G, H, L)$ is a contracting triple and let $\left(F_{n}\right)$ be any sequence of finite subsets of $L$. Let $(\mathcal{H}, \pi)$ denote the left regular representation of $G$ on $\ell^{2}(G / H)$ and let $v_{o}$ denote the indicator function of the identity coset in $G / H$. We note that $v_{o}$ is a $\pi(H)$-invariant unit vector in $\mathscr{H}$ and since $H$ has infinite index in $L$ there are no non-zero $\pi(L)$-invariant vectors in the Hilbert space $\ell^{2}(G / H)$. By the contraction property of the triple $(G, H, L)$, we can find a sequence $\left(s_{n}\right)$ in $G$ such that the inclusions $s_{n}^{-1} F_{n} s_{n} \subset H$ hold for all $n$ and thus

$$
\frac{1}{\left|F_{n}\right|} \sum_{s \in F_{n}} \pi\left(s s_{n}\right) v_{o}=\pi\left(s_{n}\right) v_{o} \text { for all } n,
$$

since $v_{o}$ is $\pi(H)$-invariant, so in particular the Hilbert norm of this expression equals one for every $n$, which shows that the pair $(G, L)$ flabby.

The following corollary now follows from Theorem 1.5 and the discussions in Section 9 ,

Corollary 1.4. The (locally finite) group $\operatorname{Sym}_{o}(\mathbb{N})$ of all permutations on $\mathbb{N}$ with finite supports is flabby, and thus the Strong Left Ergodic Theorem fails for this group.

1.9. Automatic pointwise ergodic theorems. The final section of this paper has as its goal to show that mean and pointwise ergodic theory of triples can exhibit dramatically different phenomena than in the classically studied "group case". This will be done by providing examples of triples for which the mean and pointwise ergodic theorem hold along any sequence of subsets.

The key concept here is that of a rigid pair, which we define as follows. 
Definition 1.7 (Rigid pairs). Let $G$ be a countable group and let $H$ be a subgroup of $G$. We say that the pair $(G, H)$ is rigid if whenever $(\mathscr{H}, \pi)$ is a unitary representation of $G$ with a no $\pi(G)$-invariant vectors, but equipped with a $\pi(H)$-invariant unit vector $v_{o}$ in $\mathscr{H}$, then

$$
\left\langle v_{o}, \pi(s) v_{o}\right\rangle=\left\{\begin{array}{cc}
1 & \text { if } s \in H \\
0 & \text { otherwise }
\end{array} ;\right.
$$

in other words, the cyclic sub-representation spanned by $v_{o}$ is isomorphic to the left regular representation of $G$ on $\ell^{2}(G / H)$.

In Section 10 we shall show that the pairs

$$
G=\mathbb{Q} \rtimes \mathbb{Q}^{*} \text { and } H=\{0\} \rtimes \mathbb{Q}^{*}
$$

and

$$
G=\mathrm{SL}_{n}(\mathbb{Q}) \times \mathrm{SL}_{n}(\mathbb{Q}) \text { and } H=\Delta_{2}\left(\mathrm{SL}_{n}(\mathbb{Q})\right),
$$

for $n \geq 2$, where $\Delta_{2}\left(\mathrm{SL}_{n}(\mathbb{Q})\right)$ denotes the diagonal subgroup in $G$, are rigid.

Our main observation concerning rigid pairs, which will be proved in Section 10, can be stated as follows.

Theorem 1.6 (Automatic Pointwise Ergodic Theorem). Let $(G, H)$ be a rigid pair and suppose that $(X, v)$ is an ergodic probability measure-preserving $G$-space and $\phi$ is a square-integrable measurable function on $X$ which is essentially $H$-invariant. Then, for every strictly increasing nested sequence $\left(F_{n}\right)$ of finite subsets of $G / H$, we have

$$
\lim _{n} \frac{1}{\left|F_{n}\right|} \sum_{s \in F_{n}} \varphi\left(s^{-1} x\right)=\int_{X} \varphi d v
$$

for almost every $x \in X$ with respect to $v$.

1.10. Organization of the paper. The paper is divided into 10 sections as follows.

In Section 2 we recall the GNS-construction associated to a positive definite function on a countable group and list a few examples of triples (mostly of the form $(G, H, G)$ for various countable groups $G$ and subgroups $H$ thereof) which have been studied extensively in the literature and to which our results apply.

In Section 3 we give a short proof of the Left Weak Ergodic Theorem, and in Section 4 we adapt F. Riesz' classical, and very elegant, argument to prove the Right Strong Ergodic Theorem to our setting.

In Section 5 we prove that the validity of the Left Strong Ergodic Theorem for a countable amenable group is equivalent to the existence of a firm strong sequence. We then apply this result in Section 6 to show that the Left Strong Ergodic Theorem holds for all countable groups with no ICC quotients.

In Section 7 we prove that counterexamples to the Left Strong Ergodic Theorem cannot stem from induced representations from "thin" subgroups, and we discuss the situation for semidirect products of groups in detail.

In Section 8 we prove that every countable group $L$ without property (T), e.g. every amenable group, embeds into a countable group $G$ so that the Left Strong Ergodic Theorem fails for the triple $(G,\{e\}, L)$. 
In Section 9 we give explicit examples of contracting triples and conjugation-thick subgroups. In particular, we show that the locally finite group $\operatorname{Sym}_{o}(\mathbb{N})$ contains proper conjugation-thick subgroups, thereby establishing Corollary 1.4 .

In Section 10 we provide examples of so called rigid pairs $(G, H)$ of groups for which pointwise ergodic theorem is valid along any strictly increasing nested sequence of subsets of the coset space $G / H$, and we prove Theorem 1.6 .

1.11. Acknowledgments. The authors wish to express their thanks to Michael Cantrell, Alex Furman, Anders Karlsson, Amos Nevo, Felix Pogorzelski and Andreas Thom for interesting and encouraging discussions. The authors would also like to thank Max Planck Institute for Mathematics, Bonn, for their hospitality.

\section{THE GNS-CONSTRUCTION AND UNITARY REPRESENTATIONS OF COSET SPACES}

We shall now give a short list of examples of pairs $(G, H)$ of countable groups, where $H$ is a subgroup of $G$, whose unitary representations with non-zero $\pi(H)$-invariant vectors have attracted some attention in the literature.

2.1. The GNS-construction. Let $G$ be a countable group. A complex-valued function $\phi: G \rightarrow \mathbb{C}$ is called positive definite if for every finite set $c_{1}, \ldots, c_{N}$ of complex numbers and for every finite set $x_{1}, \ldots, x_{N}$ in $G$, we have

$$
\sum_{i, j=1}^{N} c_{i} \bar{c}_{j} \phi\left(x_{i}^{-1} x_{j}\right) \geq 0
$$

We note that if $(\mathscr{H}, \pi)$ is a unitary representation of $G$ and $v$ is any vector in $\mathscr{H}$, then the function

$$
\phi_{v}(x)=\langle v, \pi(x) v\rangle \quad \text { for } x \in G
$$

is positive definite on $G$. Furthermore, if $v$ is $\pi(H)$-invariant for some subgroup $H<G$, then $\phi_{v}$ is bi- $H$-invariant.

Conversely, if $H$ is a subgroup of $G$ and $\phi$ is a positive definite function on $G$ which is bi- $H$ invariant and normalized so that $\phi(e)=1$, then this function must be of the form $\phi_{v}$ for some unitary representation $\left(\mathscr{H}_{\phi}, \pi_{\phi}\right)$ of $G$ with a $\pi(H)$-invariant (unit) vector $v$. To see this, we first note that we can equip the linear space $C_{o}(G / H)$ of all complex-valued finitely supported functions on the coset space $G / H$ with the positive definite inner product

$$
\left\langle c, c^{\prime}\right\rangle_{\phi}=\sum_{x, y \in G / H} c(x) \overline{c^{\prime}(y)} \phi\left(x^{-1} y\right), \quad c, c^{\prime} \in C_{o}(G / H) .
$$

One readily checks that the left regular representation of $G$ on $C_{o}(G / H)$ is unitary with respect to the inner product $\langle\cdot, \cdot\rangle_{\phi}$. If we quotient out the (invariant) linear subspace of all elements $c$ which satisfy $\langle c, c\rangle_{\phi}=0$, and complete, then we have constructed a Hilbert space $\mathscr{H}_{\phi}$ and a unitary representation $\pi_{\phi}$ on $\mathscr{H}_{\phi}$ with a $\pi_{\phi}(H)$-invariant (cyclic) unit vector $v$ (which correspond to the equivalence class of the indicator function of the identity coset in $G / H$ ) such that

$$
\phi(H g H)=\langle v, \pi(g) v\rangle_{\phi}, \quad \text { for all } H g H \in H \backslash G / H .
$$

In the literature, the unitary representation $\left(\mathscr{H}_{\phi}, \pi_{\phi}\right)$ is often referred to as the GNS-construction of the positive definite function $\phi$.

We note that the GNS-construction associated to the positive definite function $\phi$ on $H \backslash G / H$ which satisfies $\phi(\mathrm{HeH})=1$ and $\phi(\mathrm{HgH})=0$ for all $\mathrm{g} \neq \mathrm{HeH}$ is isomorphic to the left regular representation of $G$ on $\ell^{2}(G / H)$, and the GNS-construction associated to the constant function 
one is simply the identity representation. These unitary representations of course exist for any countable group $G$ and subgroup $H$ thereof. If we want to construct other unitary representations, we should impose more conditions on the pair $(G, H)$.

2.2. Higher order characters. Let $G_{o}$ be a countable group and define for $k \geq 2$, the pair

$$
G=G_{o}^{k} \text { and } H=\Delta_{k}\left(G_{o}\right),
$$

where the latter group denotes the diagonal subgroup in $G_{o}^{k}$. We note that the coset space $G / H$ can be identified with $G_{o}^{k-1}$, and if $k=2$, then a bi- $H$-invariant positive definite function on $G$ is nothing else than a conjugation-invariant positive definite function on $G_{o}$ (often called a character).

The study of characters on countable (non-abelian) discrete groups is quite subtle in general and complete classifications are only known in a hand-full of cases. However, this research area has recently experienced a rejuvenation with a series of breakthrough results and observations; see for instance the papers [9], [21] and [26].

The cases when $k \geq 3$ seem to have attracted much less attention.

2.3. Semidirect products. Let us now consider the case when

$$
G=N \rtimes \Lambda \text { and } H=\{o\} \rtimes \Lambda,
$$

where $N$ is a countable abelian group and $\Lambda<\operatorname{Aut}(N)$. The multiplication on $G$ can be encoded on the direct product $N \times \Lambda$ by

$$
\left(n_{1}, \lambda_{1}\right)\left(n_{2}, \lambda_{2}\right)=\left(n_{1}+\lambda_{1}\left(n_{2}\right), \lambda_{1} \lambda_{2}\right) \quad \text { for all }\left(n_{1}, \lambda_{1}\right),\left(n_{2}, \lambda_{2}\right) \in N \times \Lambda .
$$

If $\phi$ is a bi- $H$-invariant positive definite function on $G$, then

$$
\phi(n, \lambda)=\phi_{o}(n) \text { for all }(n, \lambda) \in G
$$

for some positive definite function $\phi_{o}$ on $N$ which is invariant under the action of $\Lambda$. Since $N$ is abelian, a classical theorem of S. Bochner (see e.g. the book [23]) now asserts that there exists a unique probability measure $v_{o}$ on the dual (compact) group $\hat{N}$ of $N$, which is invariant under the dual action of $\Lambda$ on $\hat{N}$, such that

$$
\phi_{o}(n)=\int_{\hat{N}} \chi(n) d v_{o}(\chi), \quad \forall n \in N
$$

Conversely, if $v_{o}$ is a probability measure on $\hat{N}$ which is invariant under the dual action of $\Lambda$, then the function $\phi(n, \lambda)=\phi_{o}(n)$ is a bi- $H$-invariant positive definite on $G$ and thus corresponds via the GNS-construction to a unitary representation of $G$ with a (cyclic) $H$-invariant vector.

The study of $\Lambda$-invariant probability measures on $\mathbb{T}^{d}$ for various (thin) subgroups $\Lambda$ of the automorphism group $\operatorname{Aut}\left(\mathbb{Z}^{d}\right)=\mathrm{GL}_{d}(\mathbb{Z})$ has attracted a lot of interest in recent years and very little is known in general.

2.4. Hecke pairs. Let $G$ be a countable group. We say that a subgroup $H<G$ is almost normal (or commensurated) if every $H$-orbit on $G / H$ is finite. In particular, every normal subgroup of $G$ is almost normal. If a subgroup $H$ is almost normal, then one often refers to $(G, H)$ as a Hecke pair.

Given a Hecke pair $(G, H)$ one can always associate a pair $(\bar{G}, \bar{H})$ (known as the Schlicting completion of $(G, H)$ ), where $\bar{G}$ is a locally compact group and $\bar{H}$ is a compact open subgroup thereof, in such a way that $G$ embeds densely in $\bar{G}$ and there is a one-to-one correspondence 
between the bi- $H$-invariant positive definite functions on $G$ and bi- $\bar{H}$-invariant continuous positive definite functions on $\bar{G}$. For instance, if

$$
G=\mathrm{PSL}_{2}(Z[1 / p]) \text { and } H=\mathrm{PSL}_{2}(\mathbb{Z}),
$$

where $p$ is a prime number, then

$$
\bar{G}=\mathrm{PSL}_{2}\left(\mathbb{Q}_{p}\right) \quad \text { and } \quad \bar{H}=\mathrm{PSL}_{2}\left(\mathbb{Z}_{p}\right) .
$$

We refer the reader to the paper [1] for a concise treatment of Hecke pairs and their Schlicting completions. In the example above, the unitary representations of $\mathrm{PSL}_{2}\left(\mathbb{Q}_{p}\right)$ with a non-zero $\mathrm{PSL}_{2}\left(\mathbb{Z}_{p}\right.$ )-invariant vector (also known as class one representations) are well-known and admit explicit descriptions; see e.g. the book [16].

\section{Proof of the Left Weak Ergodic Theorem}

The proof of Theorem 1.1 is not hard and we include a proof only for completeness. Let $(G, H, L)$ be a left amenable triple and suppose that $\left(F_{n}\right)$ is a a left Følner sequence for this triple. We wish to show that if $(\mathscr{H}, \pi)$ is a (separable) unitary representation with a $\pi(H)$ invariant unit vector $v$, then

$$
\lim _{n} \frac{1}{\left|F_{n}\right|} \sum_{s \in F_{n}} \pi(s) v=P_{L} v
$$

in the weak topology on $\mathscr{H}$. Since the unit ball in $\mathscr{H}$ is weakly sequentially compact, it suffices to prove that whenever $\left(n_{j}\right)$ is a sequence such that the limit of the sequence

$$
v_{j}=\frac{1}{\left|F_{n_{j}}\right|} \sum_{s \in F_{n_{j}}} \pi(s) v
$$

exists in the weak topology, then this weak limit is $\pi(L)$-invariant, or equivalently, the difference $\pi(t) v_{j}-v_{j}$ converges to zero in the weak topology for every $t \in L$.

We readily check that

$$
\left\|\pi(t) v_{j}-v_{j}\right\|=\left\|\frac{1}{\left|F_{n_{j}}\right|} \sum_{s \in F_{n_{j}} \Delta t F_{n_{j}}} \pi(s) v\right\| \leq \frac{\left|F_{n_{j}} \Delta t F_{n_{j}}\right|}{\left|F_{n_{j}}\right|},
$$

and the right hand side tends to zero with $j$ by the left Følner property for $\left(F_{n}\right)$, which finishes the proof.

\section{Proof of the Right Strong Ergodic Theorem}

The aim of this section is to give a proof of Theorem 1.2 based on an adaption of the elegant argument of F. Riesz outlined in [22].

The main part of F. Riesz argument is an important observation about decompositions of unitary representations which in the case of triples we have adapted as follows.

Proposition 4.1 (Orthogonal splitting). Let $G$ be a countable group and suppose that $H$ and $L$ are subgroups of $G$ with $H<L<N_{G}(H)$. If $(\mathscr{H}, \pi)$ is a unitary representation of $G$, then

$$
\mathscr{H}^{H}=\mathscr{H}^{L} \oplus \operatorname{Cobnd}_{(L, H)}(\mathscr{H}, \pi)
$$

where

$$
\operatorname{Cobnd}_{(L, H)}(\mathscr{H}, \pi)=\overline{\operatorname{span}\left\{v-\pi(s) v: s \in L, v \in \mathscr{H}^{H}\right\}} \subset \mathscr{H}^{H} .
$$


Once this result has been established, the proof of Theorem 1.2 is immediate. We recall that we wish to prove that if $\left(F_{n}\right)$ is a right Følner sequence for a right amenable triple $(G, H, L)$ and $(\mathscr{H}, \pi)$ is a unitary representation of $G$ with a $\pi(H)$-invariant unit vector $v$, then

$$
\lim _{n} \frac{1}{\left|F_{n}\right|} \sum_{s \in F_{n}} \pi(s) v=P_{L} v
$$

in the norm topology on $\mathscr{H}$. We note that it is enough to prove this for a set of vectors $v$ in $\mathscr{H}$ which span a norm-dense subspace of $\mathscr{H}$. The proposition above asserts that the set

$$
S=\left\{v+w-\pi\left(s_{o}\right) w: v \in \mathscr{H}^{L}, w \in \mathscr{H}^{H} \text { and } s_{o} \in L\right\}
$$

spans a norm-dense subspace of $\mathscr{H}$, and

$$
\left\|\frac{1}{\left|F_{n}\right|} \sum_{s \in F_{n}}\left(\pi(s) v+\pi(s) w-\pi\left(s s_{o}\right) w\right)-v\right\| \leq \frac{\left|F_{n} \triangle \alpha_{H}\left(s_{o}\right) F_{n}\right|}{\left|F_{n}\right|} \rightarrow 0,
$$

as $n$ tends to infinity, since $\pi(s) v=v$ for all $s \in F_{n} \subset L / H$ and $P_{L}\left(v+w-\pi\left(s_{o}\right) w\right)=v$ and $\left(F_{n}\right)$ is a right Følner sequence for $(G, H, L)$, which finishes the proof of Theorem 1.2 .

Proof of Proposition 4.1 We note that it suffices to show that if $v \in \mathscr{P}^{H}$ is orthogonal to all elements of the form $w-\pi(s) w$ with $w \in \mathscr{H}^{H}$ and $s \in L$, then $w$ is $\pi(L)$-invariant. Since

$$
\langle v, w-\pi(s) w\rangle=\left\langle v-\pi\left(s^{-1}\right) v, w\right\rangle=0 \quad \text { for all } w \in \mathscr{P}^{H} \text { and } s \in L,
$$

and

$$
v-\pi\left(s^{-1}\right) v \in \mathscr{H}^{H} \quad \text { for all } s \in L<N_{G}(H),
$$

we conclude that $v=\pi(s) v$ for all $s \in L$.

\section{Proof of the Transfer Principle}

We note that if the Left Strong Ergodic Theorem holds for a countable amenable group $G$, then every left Følner sequence in $G$ is a firm strong sequence, so to prove Proposition [1.1 it suffices to establish the following "local" transfer principle:

Proposition 5.1 (Local transfer principle). Let $G$ be a countable amenable group and let $(\mathscr{H}, \pi)$ be a unitary representation of $G$. Suppose that there exists at least one firm strong sequence (not necessarily a left Følner sequence) in $G$ with respect to $(\mathcal{H}, \pi)$. Then the Left Strong Ergodic Theorem holds for $(\mathcal{H}, \pi)$.

We shall begin by reducing the proof of the proposition to a general lemma about left Følner sequences in amenable groups which should be interesting in its own right.

Suppose that we are given a unit vector $v \in \mathscr{H}$ and define the bounded real-valued function

$$
\phi(s, t)=\Re\langle\pi(s) v, \pi(t) v\rangle, \quad \text { for }(s, t) \in G \times G,
$$

where $\Re$ denotes the real part of a complex number.

Let us now suppose that $\left(K_{m}^{o}\right)$ is a firm strong sequence in $G$ with respect to the unitary representation $(\mathscr{H}, \pi)$ of $G$. We note that for every pair of sequences $\left(s_{m}^{o}\right)$ and $\left(t_{m}^{o}\right)$ in $G$,

$$
\begin{aligned}
\left|\frac{1}{\left|K_{m}^{o}\right|^{2}} \sum_{(s, t) \in K_{m}^{o} \times K_{m}^{o}} \phi\left(s s_{m}^{o}, t t_{m}^{o}\right)\right| & =\left|\Re\left\langle\frac{1}{\left|K_{m}^{o}\right|} \sum_{s \in K_{m}^{o}} \pi\left(s s_{m}^{o}\right) v, \frac{1}{\left|K_{m}^{o}\right|} \sum_{t \in K_{m}^{o}} \pi\left(t t_{m}^{o}\right) v\right\rangle\right| \\
& \leq\left\|\frac{1}{\left|K_{m}^{o}\right|} \sum_{s \in K_{m}^{o}} \pi\left(s s_{m}^{o}\right) v\right\| \rightarrow 0 .
\end{aligned}
$$


We wish to prove that if $\left(F_{n}^{o}\right)$ is a left Følner sequence in $G$, then

$$
\varlimsup_{n}\left\|\frac{1}{\left|F_{n}^{o}\right|} \sum_{s \in F_{n}^{o}} \pi(s) v\right\|^{2}=\varlimsup_{n} \frac{1}{\left|F_{n}^{o}\right|^{2}} \sum_{(s, t) \in F_{n}^{o} \times F_{n}^{o}} \phi(s, t)=0,
$$

where the first equality sign stems from

$$
\sum_{(s, t) \in F_{n}^{o} \times F_{n}^{o}}\langle\pi(s) v, \pi(t) v\rangle=\sum_{(s, t) \in F_{n}^{o} \times F_{n}^{o}} \frac{1}{2}(\langle\pi(s) v, \pi(t) v\rangle+\langle\pi(t) v, \pi(s) v\rangle)=\sum_{(s, t) \in F_{n}^{o} \times F_{n}^{o}} \phi(s, t),
$$

since the inner product on $\mathscr{H}$ is skew-hermitian.

Proposition 1.1 is now an immediate consequence of the following lemma (which is a variation of Lemma 3.3 in [3]) applied to the (amenable) direct product group $G \times G$, the sequences

$$
K_{m}=K_{m}^{o} \times K_{m}^{o} \quad \text { and } \quad F_{n}=F_{n}^{o} \times F_{n}^{o},
$$

in $G \times G$ and the bounded real-valued function $\phi$ on $G \times G$ defined above. We note that $\left(F_{n}\right)$ is a left Følner sequence in $G \times G$.

Lemma 5.1. Let $G$ be a countable amenable group and fix a left $F \varnothing l n e r$ sequence $\left(F_{n}\right)$ in $G$ and a bounded real-valued function $\phi$ on $G$. Then, for every sequence $\left(K_{m}\right)$ of finite subsets of $G$, there exists a sequence $\left(t_{m}\right)$ in $G$ such that

$$
\varlimsup_{n} \frac{1}{\left|F_{n}\right|} \sum_{t \in F_{n}} \phi(t) \leq \frac{\lim }{m} \frac{1}{\left|K_{m}\right|} \sum_{s \in K_{m}} \phi\left(s t_{m}\right) .
$$

Proof. We extract a subsequence $\left(n_{j}\right)$ such that

$$
\beta=\varlimsup_{n} \frac{1}{\left|F_{n}\right|} \sum_{t \in F_{n}} \phi(t)=\lim _{j} \frac{1}{\left|F_{n_{j}}\right|} \sum_{t \in F_{n_{j}}} \phi(t) .
$$

Since $\left(F_{n_{j}}\right)$ is a left Følner sequence in $G$ and $\phi$ is bounded and real-valued, we can find, for every integer $m$, an index $j$ such that

$$
\frac{1}{\left|F_{n_{j}}\right|} \sum_{t \in F_{n_{j}}} \phi(s t) \geq \beta-\frac{1}{m}
$$

for all $s \in K_{m}$, and thus

$$
\frac{1}{\left|K_{m}\right|} \sum_{s \in K_{m}}\left(\frac{1}{\left|F_{n_{j}}\right|} \sum_{t \in F_{n_{j}}} \phi(s t)\right)=\frac{1}{\left|F_{n_{j}}\right|} \sum_{t \in F_{n_{j}}}\left(\frac{1}{\left|K_{m}\right|} \sum_{s \in K_{m}} \phi(s t)\right) \geq \beta-\frac{1}{m} .
$$

In particular, there must exist at least one element $t_{m} \in F_{n_{j}}$ such that

$$
\frac{1}{\left|K_{m}\right|} \sum_{s \in K_{m}} \phi\left(s t_{m}\right) \geq \beta-\frac{1}{m},
$$

which shows that

$$
\frac{\lim }{m} \frac{1}{\left|K_{m}\right|} \sum_{s \in K_{m}} \phi\left(s t_{m}\right) \geq \beta
$$




\section{A Left Strong ERgodic Theorem For nilpotent groups}

The aim of this section is to prove Theorem 1.3, but before we can do this we need to show that every countable group $G$ without ICC quotients can be decomposed as a (possibly) transfinite increasing chain of normal subgroups $\left(G_{\alpha}\right)$ so that for every unitary representation $(\mathscr{H}, \pi)$ of the group $G$, the Left Strong Ergodic Theorem is immediate for elements in the unitary sub-representation $\operatorname{Cobnd}_{\left(G, G_{\alpha}\right)}(\mathcal{H}, \pi)$ for every $\alpha$. By a rather straightforward approximation argument, this yields the Left Strong Ergodic Theorem for the whole group $G$.

Let $G$ be a countable group and let $H$ be a normal subgroup of $G$. We denote by $p_{H}$ the canonical quotient homomorphism from $G$ onto $G / H$, and for $x \in G$ we write $x^{G}$ for the conjugation class of $x$, i.e. the set of all conjugates of $x$ in $G$. We define

$$
\mathrm{FC}_{G}(H)=\left\{x \in G: p_{H}\left(x^{G}\right) \subset G / H \text { is finite }\right\},
$$

and we note that since $H$ is normal, $\mathrm{FC}_{G}(H)$ is again a normal subgroup of $G$. In particular, if $H$ is the trivial subgroup, then $\mathrm{FC}_{G}(H)$ consists of exactly those elements in $G$ with a finite conjugation class.

We can also iterate this construction: $\operatorname{Set~}_{\mathrm{FC}_{G}^{1}}(H)=\mathrm{FC}_{G}(H)$ and for every integer $k \geq 1$, we define

$$
\mathrm{FC}_{G}^{(k+1)}(H)=\mathrm{FC}_{G}\left(\mathrm{FC}_{G}^{(k)}(H)\right) .
$$

More generally, if $\alpha$ is an ordinal, then we define

$$
\mathrm{FC}_{G}^{(\alpha+1)}(H)=\mathrm{FC}_{G}\left(\mathrm{FC}_{G}^{(\alpha)}(H)\right) \text { and } \mathrm{FC}^{(\alpha)}(G)=\mathrm{FC}_{G}^{(\alpha)}(\{e\}) .
$$

We note that $\mathrm{FC}_{G}^{(\alpha)}(H) \subset \mathrm{FC}_{G}^{(\alpha+1)}(H)$ for every ordinal $\alpha$, so if $\lambda$ is a limit ordinal, then it is well-defined to set

$$
\mathrm{FC}_{G}^{(\lambda)}(H)=\bigcup_{\alpha<\lambda} \mathrm{FC}_{G}^{(\alpha)}(H)
$$

Since $G$ is a countable, the least ordinal $\alpha_{H}$ such that

$$
\mathrm{FC}_{G}^{\left(\alpha_{H}+1\right)}(H)=\mathrm{FC}_{G}^{\left(\alpha_{H}\right)}(H)
$$

is countable, and we set $G_{\text {icc }}=\mathrm{FC}^{\alpha^{\{e\}}}(G)$. We note that every non-identity element (if any) in the quotient group $G / G_{\text {icc }}$ must have an infinite conjugation class, so we have thus proved the following proposition.

Proposition 6.1 (Characterization of ICC quotients). A countable group G does not admit any ICC quotients if and only if there exists an ordinal $\alpha$ such that $\mathrm{FC}^{\alpha_{[e\}}}(G)=G$.

Remark 6.1. We note that every countable group all of whose conjugation classes are finite is amenable. Indeed, if $L$ is such a group, then there exists an increasing exhaustion $\left(F_{n}\right)$ of $L$ by finite conjugation-invariant subsets. Hence we can identify the image of the conjugation action map $p: L \rightarrow \operatorname{Sym}(L)$ with the increasing union of the finite groups $\operatorname{Sym}\left(F_{n}\right)$, which is clearly amenable. Since the kernel of the action map $p$ equals the center of $L$ (which is abelian), we conclude that $L$ is amenable.

Now, if $G$ is any countable group and $H$ is a normal subgroup of $G$, then $\operatorname{FC}_{G}(H) / H$ is clearly isomorphic to $\mathrm{FC}_{G / H}(\{e\})$, which is amenable by the discussion above, and thus $\mathrm{FC}_{G}(H)$ is amenable whenever $H$ is amenable.

Example (Nilpotent groups). We recall that a countable group is nilpotent if its upper central series terminates after a finite number of steps, that is to say, if we define $Z_{o}=\{e\}$ and

$$
Z_{i+1}=\left\{s \in G:[s, t] \in Z_{i} \text { for all } t \in G\right\},
$$


then there exists an integer $k$ such that

$$
\{e\}=Z_{o} \triangleleft Z_{1} \triangleleft \ldots \triangleleft Z_{k}=G .
$$

Since $Z_{i} \subset \mathrm{FC}^{(i)}(G)$ for every $i$, we have $G=\mathrm{FC}^{(k)}(G)$ as well. More generally, we say that $G$ is virtually nilpotent if it contains a nilpotent subgroup $G_{o}$ of finite index. We leave it as an exercise to verify that $\mathrm{FC}^{(k)}(G)=G$ whenever $\mathrm{FC}^{(k)}\left(G_{o}\right)=G_{o}$, and thus virtually nilpotent groups do not admit any ICC quotients either.

We shall now turn to the proof of Theorem 1.3, Let $G$ be a countable group which does not admit any ICC quotient groups and suppose that $(\mathscr{H}, \pi)$ is a unitary representation of $G$. By Proposition 4.1 we can write

$$
\mathscr{H}=\mathscr{P}^{G_{1}} \oplus \operatorname{Cobnd}_{\left(G_{1}, G_{o}\right)}(\mathscr{H}, \pi)=\ldots=\mathscr{P}^{G_{k-1}} \oplus\left(\bigoplus_{i=0}^{k-2} \operatorname{Cobnd}_{\left(G_{i+1}, G_{i}\right)}(\mathscr{H}, \pi)\right)
$$

for every $k$, where $G_{i}=\mathrm{FC}^{(i)}(G)$. For a fixed integer $k$, we set

$$
\mathscr{H}_{k}=\bigoplus_{i=0}^{k-2} \operatorname{Cobnd}_{\left(G_{i+1}, G_{i}\right)}(\mathscr{H}, \pi)
$$

and define inductively for any ordinal $\alpha$,

$$
\mathscr{H}_{\alpha+1}=\mathscr{H}_{\alpha} \oplus \operatorname{Cobnd}_{\left(G_{\alpha+1}, G_{\alpha}\right)}(\mathscr{H}, \pi)
$$

If $\lambda$ is a limit ordinal, we set

$$
\mathscr{H}_{\lambda}=\overline{\bigcup_{\alpha<\lambda} \mathscr{H}_{\alpha}} \subset \mathscr{H}
$$

If we assume that the representation $(\mathscr{H}, \pi)$ does not have any non-zero $\pi(G)$-invariant vectors, then $\mathscr{H}=\mathscr{H}_{\lambda}$, where $\lambda$ is the smallest ordinal such that $G=G_{\lambda}$.

We wish to prove that if $\left(F_{n}\right)$ is any right Følner sequence in $G$ and $\left(s_{n}\right)$ is any sequence in $G$, then

$$
\varlimsup_{n}\left\|\frac{1}{\left|F_{n}\right|} \sum_{s \in F_{n}} \pi\left(s s_{n}\right) v\right\|=0, \quad \text { for all } v \in \mathscr{H}
$$

We note that it is enough to establish these limits for a dense subspace of $\mathscr{H}$, so in particular we only need to calculate the limit above for vectors of the form $v_{\alpha}-\pi\left(t_{\alpha}\right) v_{\alpha}$, where $v_{\alpha}$ belongs to $\mathscr{P}^{G_{\alpha}}$ and $t_{\alpha}$ is in $G_{\alpha+1}$ for some ordinal $\alpha$.

We fix a vector of this form and assume that (6.1) fails for some sequence $\left(s_{n}\right)$, that is to say, possibly upon passing to a subsequence, we assume that

$$
\lim _{n}\left\|\frac{1}{\left|F_{n}\right|} \sum_{s \in F_{n}}\left(\pi\left(s s_{n}\right) v_{\alpha}-\pi\left(s s_{n}\right) \pi\left(t_{\alpha}\right) v_{\alpha}\right)\right\|>0 .
$$

We recall that since $t_{\alpha} \in G_{\alpha+1}$, the set

$$
S_{\alpha}=\left\{p_{G_{\alpha}}\left(s_{n} t_{\alpha} s_{n}^{-1}\right): n \geq 1\right\} \subset G / G_{\alpha}
$$

is finite, and thus, possibly upon passing to a further subsequence, we may assume that the identities $s_{n} t_{\alpha} s_{n}^{-1}=u_{\alpha}$ hold modulo $G_{\alpha}$ for all $n$ and for some element $u_{\alpha} \in G_{\alpha+1}$. Hence, along this subsequence, we have

$$
\frac{1}{\left|F_{n}\right|} \sum_{s \in F_{n}}\left(\pi\left(s s_{n}\right) v_{\alpha}-\pi\left(s s_{n}\right) \pi\left(t_{\alpha}\right) v_{\alpha}\right)=\frac{1}{\left|F_{n}\right|} \sum_{s \in F_{n}}\left(\pi(s)-\pi\left(s u_{\alpha}\right)\right) \pi\left(s_{n}\right) v_{\alpha}
$$


since $v_{\alpha}$ is $G_{\alpha}$-invariant, and we conclude that

$$
\left\|\frac{1}{\left|F_{n}\right|} \sum_{s \in F_{n}}\left(\pi(s)-\pi\left(s u_{\alpha}\right)\right) \pi\left(s_{n}\right) v_{\alpha}\right\|=\left\|\frac{1}{\left|F_{n}\right|} \sum_{s \in F_{n} \triangle F_{n} u_{\alpha}} \pi\left(s s_{n}\right) v_{\alpha}\right\| \leq \frac{\left|F_{n} \triangle F_{n} u_{\alpha}\right|}{\left|F_{n}\right|} \rightarrow 0,
$$

since $\left(F_{n}\right)$ is a right Følner sequence in $G$. This contradiction finishes the proof of Theorem 1.3 .

In fact, the arguments above also yield the following strengthening of Theorem 1.3.

Proposition 6.2. Let $G$ be a countable amenable group and suppose that $(\mathscr{H}, \pi)$ is a unitary representation of $G$. Then there exists a normal subgroup $G_{i c c}$ of $G$ with the property that the quotient group $G / G_{i c c}$ is either trivial or ICC and a closed $\pi(G)$-invariant subspace $\mathscr{H}_{0} \subset \mathscr{H}$ such that

$$
\mathscr{H}=\mathscr{H}^{G_{i c c}} \oplus \mathscr{H}_{o}
$$

and the Left Strong Ergodic Theorem holds for $\left(\mathscr{H}_{0}, \pi\right)$.

6.1. An outline of the proof of Corollary 1.3, Let $G$ be a countable amenable group and assume that $(\mathscr{H}, \pi)$ is a unitary representation of $G$ with no non-zero $\pi(G)$-invariant vectors. By Proposition 6.2 there exists a closed $\pi(G)$-invariant subspace $\mathscr{H}_{0}$ of $\mathscr{H}$ so that the Left Strong Ergodic Theorem holds for $\left(\mathscr{H}_{0}, \pi\right)$ and

$$
\mathscr{H}=\mathscr{H}^{G_{\text {icc }}} \oplus \mathscr{H}_{o},
$$

where $G_{i c c}<G$ is a normal subgroup with the property that the quotient group $G / G_{i c c}$ is either trivial or ICC.

In particular, if we assume that there exists a left Følner sequence $\left(F_{n}\right)$ in $G$ such that the Left Strong Ergodic Theorem fails for $(\mathscr{H}, \pi)$ along this sequence, then we can conclude that $G_{\text {icc }} \neq G$ and that the Left Strong Ergodic Theorem fails for $\left(\mathscr{P}^{G_{i c c}}, \pi\right)$ along the sequence $\left(F_{n}\right)$. That is to say, for some unit vector $v \in \mathscr{H}^{G_{i c c}}$, we have

$$
\delta=\varlimsup_{n}\left\|\frac{1}{\left|F_{n}\right|} \sum_{s \in F_{n}} \pi(s) v\right\|>0 .
$$

Clearly, the unitary representation $\left(\mathscr{P}^{G_{i c c}}, \pi\right)$ of $G$ factors through a unitary representation $\pi^{\prime}$ on $\mathscr{H}^{\prime}=\mathscr{H}^{G_{i c c}}$ of the quotient group $G / G_{i c c}$, and we can write

$$
\frac{1}{\left|F_{n}\right|} \sum_{s \in F_{n}} \pi(s) v=\sum_{t \in G / G_{i c c}} \beta_{n}(t) \pi^{\prime}(t) v
$$

where

$$
\beta_{n}\left(t G_{i c c}\right)=\frac{1}{\left|F_{n}\right|} \sum_{s \in G_{i c c}} \chi_{F_{n}}(t s), \quad \text { for all } t G_{i c c} \in G / G_{i c c} .
$$

One can readily verify that $\left(\beta_{n}\right)$ satisfies

$$
\lim _{n} \sum_{t \in G / G_{i c c}}\left|\beta_{n}(u t)-\beta_{n}(t)\right|=0 \quad \text { for all } u \in G / G_{i c c},
$$

and

$$
\varlimsup_{n} \sum_{s, t \in G / G_{i c c}} \beta_{n}(s) \beta_{n}(t) \Re\left\langle\pi^{\prime}(s) v, \pi^{\prime}(t) v\right\rangle=\delta^{2}>0,
$$

where $\Re$ denotes the real part of a complex number.

In particular, any accumulation point $\lambda$ of the sequence

$$
\beta_{n} \otimes \beta_{n} \in \ell^{1}\left(G / G_{i c c} \times G / G_{i c c}\right) \subset \ell^{1}\left(G / G_{i c c} \times G / G_{i c c}\right)^{* *}
$$


is invariant under left translations by elements in the direct product group $G / G_{i c c} \times G / G_{i c c}$. These limits are often referred to as left invariant means on $G / G_{i c c} \times G / G_{i c c}$, and we note that

$$
\lambda(\phi) \geq \frac{1}{2} \cdot \delta^{2}
$$

where $\phi$ is the bounded real-valued function on $G / G_{i c c} \times G / G_{i c c}$ defined by

$$
\phi(s, t)=\Re\left\langle\pi^{\prime}(s) v, \pi^{\prime}(t) v\right\rangle, \quad \text { for all }(s, t) \in G / G_{i c c} \times G / G_{i c c} .
$$

We stress that this expression is well-defined since $v$ is assumed to be invariant under $G_{i c c}$.

A classical argument by I. Namioka in [19] will now guarantee that we can find a left Følner sequence $\left(L_{n}\right)$ in $G / G_{i c c} \times G / G_{i c c}$ such that

$$
\varlimsup_{n} \frac{1}{\left|L_{n}\right|} \sum_{(s, t) \in L_{n}} \phi(s, t) \geq \frac{1}{2} \cdot \delta^{2} .
$$

Let $\left(F_{n}^{\prime}\right)$ be any left Følner sequence in $G / G_{i c c}$. By Lemma 5.1 we can now find a sequence of elements $\left(s_{n}, t_{n}\right)$ in the direct product group $G / G_{i c c} \times G / G_{i c c}$ such that

$$
0<\frac{1}{2} \cdot \delta^{2} \leq \varlimsup_{n} \frac{1}{\left|L_{n}\right|} \sum_{(s, t) \in L_{n}} \phi(s, t) \leq \frac{\lim }{n} \frac{1}{\left|F_{n}^{\prime}\right|^{2}} \sum_{(s, t) \in F_{n}^{\prime} \times F_{n}^{\prime}} \phi\left(s s_{n}, t t_{n}\right) .
$$

Assume that the Left Strong Ergodic Theorem holds for the unitary representation $\left(\mathscr{P}^{G_{i c c}}, \pi^{\prime}\right)$ of $G / G_{i c c}$. Then,

$$
\lim _{n} \frac{1}{\left|F_{n}^{\prime}\right|} \sum_{s \in F_{n}^{\prime}} \pi\left(s s_{n}\right) v=0 \quad \text { and } \quad \lim _{n} \frac{1}{\left|F_{n}^{\prime}\right|} \sum_{s \in F_{n}^{\prime}} \pi\left(s t_{n}\right) v=0
$$

in the norm topology on $\mathscr{H}$, which implies that

$$
\frac{1}{\left|F_{n}^{\prime}\right|^{2}} \sum_{(s, t) \in F_{n}^{\prime} \times F_{n}^{\prime}} \phi\left(s s_{n}, t t_{n}\right) .=\left\langle\frac{1}{\left|F_{n}^{\prime}\right|} \sum_{s \in F_{n}^{\prime}} \pi^{\prime}\left(s s_{n}\right) v, \frac{1}{\left|F_{n}^{\prime}\right|} \sum_{s \in F_{n}^{\prime}} \pi^{\prime}\left(s t_{n}\right) v\right\rangle=0 .
$$

This contradiction shows that the Left Strong Ergodic Theorem for $\left(\mathscr{P}^{G_{i c c}}, \pi^{\prime}\right)$ must fail at least along one of the left Følner sequences $\left(F_{n}^{\prime} s_{n}\right)$ or $\left(F_{n}^{\prime} t_{n}\right)$, and finishes the proof of Corollary 1.3.

\section{A Left Strong ERgodic Theorem For INDUCED REPRESENTATions}

The aim of this section is to prove Proposition 1.3 . We begin by recalling some basic properties of induced representations.

Let $G$ be a countable group. If $\left(\mathscr{H}_{o}, \pi_{o}\right)$ is a unitary representation of a subgroup $L<G$, one can induce a unitary representation of $G$ from $\left(\mathscr{H}_{o}, \pi_{o}\right)$ as follows. We define the linear space

$$
\mathscr{H}^{\prime}=\left\{f: G \rightarrow \mathscr{H}_{o}:\|f\|<\infty \quad \text { and } \quad f(l g)=\pi_{o}(l)^{-1} f(g), \quad \text { for all } l \in L \text { and } g \in G\right\} .
$$

Here, $\|\cdot\|$ denotes the norm induced from the following inner product

$$
\left\langle f, f^{\prime}\right\rangle=\sum_{x \in L \backslash G}\left\langle f(x), f^{\prime}(x)\right\rangle_{o},
$$

on $\mathscr{H}$, where $\langle\cdot, \cdot\rangle_{o}$ is the inner product on $\mathscr{H}_{0}$. We readily check that $\mathscr{H}^{\prime}$ is a pre-Hilbert space and we denote by $\mathscr{H}$ the Hilbert space completion on $\mathscr{H}^{\prime}$. We note that $G$ acts on $\mathscr{H}^{\prime}$ (and thus on $\mathscr{H}$ ) via the representation

$$
(\pi(g) f)(x)=f(x g), \quad \text { for } g, x \in G,
$$


which is unitary with respect to $\langle\cdot, \cdot\rangle$. This representation is often referred to as the induced representation of $\left(\mathscr{H}_{o}, \pi_{o}\right)$ and is commonly denoted by $\operatorname{Ind}_{L}^{G} \pi_{o}$.

Suppose that we are given a sequence $\left(F_{n}\right)$ of finite subsets of $G$. We wish to prove that if $L$ is thin with respect to $\left(F_{n}\right)$, by which we mean that

$$
\lim _{n} \sup _{x, y \in G / L} \frac{\left|F_{n} \cap x L y^{-1}\right|}{\left|F_{n}\right|}=0
$$

then $\left(F_{n}\right)$ is a firm strong sequence with respect to the induced representation $(\mathscr{H}, \pi)$, that is to say, for every sequence $\left(s_{n}\right)$ in $G$, we have

$$
\lim _{n} \frac{1}{\left|F_{n}\right|} \sum_{s \in F_{n}} \pi\left(s s_{n}\right) v=0
$$

in the norm topology on $G$. The assumption that the right hand side above should vanish is justified once we notice that (7.1) forces the index of $L$ in $G$ to be infinite and that induced representations from an infinite index subgroup $L$ never have non-zero $\pi(G)$-invariant vectors.

The following lemma is quite standard and we give the proof for completeness.

Lemma 7.1. For every sequence of orthogonal vectors $\left(v_{k}\right)$ in $\mathscr{H}_{o}$ such that

$$
\mathscr{H}_{o}=\bigoplus_{k} \overline{\operatorname{span}\left\{\pi_{o}(l) v_{k}: l \in L\right\}}
$$

we have

$$
\mathscr{H}=\bigoplus_{k} \overline{\operatorname{span}\left\{\pi(g) f_{k}: g \in G\right\}}
$$

where the function $f_{k}: G \rightarrow \mathscr{H}_{o}$ is defined by

$$
f_{k}(x)=\left\{\begin{array}{cl}
\pi_{o}(x)^{-1} v_{k} & \text { if } x \in L \\
0 & \text { if } x \notin L
\end{array} .\right.
$$

In particular, if $\left(\mathscr{H}_{0}, \pi_{0}\right)$ is cyclic, then so is $(\mathscr{H}, \pi)$.

Proof. We note that once we have checked that $f_{k}$ is indeed an element in the induced representation $\mathscr{H}$ for every $k$, then it suffices to show that if $f \in \mathscr{H}$ satisfies

$$
\left\langle f, \pi(s) f_{k}\right\rangle=0 \text { for all } s \in G,
$$

and for every index $k$, then $f(s)$ is zero in $\mathscr{H}_{o}$ for every $s \in G$. Upon expanding the inner product, we see that this condition exactly means that

$$
\left\langle f(s), v_{k}\right\rangle_{o}=0 \quad \text { for all } s \in G \text { and for every } k,
$$

since $f_{k}$ vanishes off $L$. In particular, for every $s \in G$, we have

$$
\left\langle f(l s), v_{k}\right\rangle_{o}=\left\langle f(s), \pi_{o}(l) v_{k}\right\rangle_{o}=0 \quad \text { for all } l \in L \text { and for every } k,
$$

which forces $f(s)=0$ for all $s \in G$, since every $v_{k}$ is cyclic for a sub-representation of $\mathscr{H}_{o}$ and together all of these sub-representation are assumed to span $\mathscr{H}_{0}$.

We now fix an orthogonal decomposition of $\mathscr{H}_{o}$ as in Lemma 7.1 with unit vectors $\left(v_{k}\right)$. We readily verify that the positive definite functions associated to the elements $\left(f_{k}\right)$ in $\mathscr{H}$ have the form:

$$
\phi_{k}(g)=\left\langle f_{k}, \pi(g) f_{k}\right\rangle=\left\{\begin{array}{cl}
\left\langle v_{k}, \pi_{o}(g) v_{k}\right\rangle_{o} & \text { if } g \in L \\
0 & \text { if } g \notin L
\end{array} .\right.
$$


It is not hard to see that if suffices to establish (7.2) for $f_{k}$ for every $k$, which explicitly means that one has to prove that for every sequence $\left(s_{n}\right)$ in $G$,

$$
\varlimsup_{n}\left\|\frac{1}{\left|F_{n}\right|} \sum_{s \in F_{n}} \pi\left(s s_{n}\right) f_{k}\right\|^{2}=\varlimsup_{n} \frac{1}{\left|F_{n}\right|^{2}} \sum_{(s, t) \in F_{n} \times F_{n}} \phi_{k}\left(s_{n}^{-1} s^{-1} t s_{n}\right)=0 .
$$

We note that in the case when $L$ is thin with respect to $\left(F_{n}\right)$, then this is a straightforward consequence of the following lemma.

Lemma 7.2. For every finite subset $F \subset G$, we have

for all $k$.

$$
\sup _{u \in G}\left|\frac{1}{|F|^{2}} \sum_{(s, t) \in F \times F} \phi_{k}\left(u^{-1} s^{-1} t u\right)\right| \leq \sup _{x, y \in G / L} \frac{\left|F \cap x L y^{-1}\right|}{|F|}
$$

Proof. We first observe that if $F \subset G$ is a finite subset of $G$, then

$$
F^{-1} F \cap L=\bigcup_{x \in G / L}(F \cap x L)^{-1}(F \cap x L),
$$

and thus, for every $u$ in $G$,

$$
\sum_{s \in F} \sum_{t \in F u}\left\langle\pi(s) f_{k}, \pi(t) f_{k}\right\rangle=\sum_{x \in G / L} \sum_{s \in F u \cap x L} \sum_{t \in F u \cap x L}\left\langle\pi(s) f_{k}, \pi(t) f_{k}\right\rangle,
$$

since $\left\langle\pi(s) f_{k}, \pi(t) f_{k}\right\rangle$ vanishes unless $s$ and $t$ belong to the same $F \cap x L$, and these sets are disjoint as $x$ ranges over $G / L$. In particular, we have

$$
\begin{aligned}
\left|\frac{1}{|F|^{2}} \sum_{s \in F} \sum_{t \in F u}\left\langle\pi(s) f_{k}, \pi(t) f_{k}\right\rangle\right| & =\left|\frac{1}{|F|^{2}} \sum_{x \in G / L} \sum_{s \in F u \cap x L} \sum_{t \in F u \cap x L}\left\langle\pi(s) f_{k}, \pi(t) f_{k}\right\rangle\right| \\
& \leq \sum_{x \in G / L}\left(\frac{|F u \cap x L|}{|F|}\right)^{2} \\
& \leq \sup _{x \in G / L} \frac{\left|F \cap x L u^{-1}\right|}{|F|} \cdot \sum_{x \in G / L} \frac{\left|F \cap x L u^{-1}\right|}{|F|} \\
& \leq \sup _{x, y \in G / L} \frac{\left|F \cap x L y^{-1}\right|}{|F|},
\end{aligned}
$$

where the first inequality sign holds since $\phi_{k}$ is bounded in absolute value by one. This finishes the proof.

7.1. Some remarks about semidirect products of groups. We end this section with a few observations about subgroups of semidirect products of groups.

We say that a countable group $G$ splits as a semidirect product if there exist two subgroups $N$ and $A$ of $G$ with $N$ normal in $G$ such that $N \cap A$ is the trivial subgroup. This means that we can write every element $g$ in $G$ uniquely as a product $g=n a$ with $n \in N$ and $a \in A$, and upon identifying $G$ with $N \times A$ via the map $(n, a) \mapsto n a$, we can encode the group multiplication in $G$ on $N \times A$ by

$$
\left(n_{1}, a_{1}\right)\left(n_{2}, a_{2}\right)=\left(n_{1} a_{1} n_{2} a_{1}^{-1}, a_{1} a_{2}\right), \quad\left(n_{1}, a_{1}\right),\left(n_{2}, a_{2}\right) \in N \times A .
$$

We note that if $N$ and $A$ are amenable groups, then so is $G$, and if $\left(B_{n}\right)$ and $\left(A_{n}\right)$ are left Følner sequences in the subgroups $N$ and $A$ respectively, such that

$$
\limsup _{n} \frac{\left|a B_{n} a^{-1} \triangle B_{n}\right|}{\left|B_{n}\right|}=0, \quad \text { for all } a \in A,
$$

then $\left(B_{n} A_{n}\right)$ is a left Følner sequence in $G$. 
We shall prove below that under some natural conditions on $N$ and $A$, the Left Strong Ergodic Theorem holds for any unitary representation of $G$ which is induced from a unitary representation of a subgroup of either $N$ or $A$.

We first prove that unitary representations induced from normal subgroups of a countable amenable group $G$ can never be counter-examples to the Left Strong Ergodic Theorem.

Proposition 7.1. Let $G$ be a countable amenable group and let $N$ be a normal subgroup of $G$ of infinite index. Then $N$ is a thin subgroup with respect to every left Følner sequence in $G$, and thus every such sequence is a firm strong sequence with respect to any unitary representation induced from $N$.

Proof. Suppose that $N$ has infinite index in $G$ and that there exists a left Følner sequence $\left(F_{n}\right)$ in $G$ such that $N$ is not thin with respect to this sequence. We shall prove that this leads to a contradiction. Since $N$ is normal in $G$, we can find (possibly upon passing to a subsequence) a sequence $\left(x_{n}\right)$ in $G$ such that

$$
\delta=\lim _{n} \frac{\left|F_{n} x_{n} \cap N\right|}{\left|F_{n}\right|}>0 .
$$

Since $\left(F_{n}\right)$ is a left Følner sequence, we can find, for every finite subset $K \subset G / N$, an index $n$ such that

$$
\frac{\left|F_{n} x_{n} \cap x N\right|}{\left|F_{n}\right|} \geq \frac{\delta}{2} \quad \text { for all } x \in K,
$$

and thus

$$
1=\frac{\left|F_{n} x_{n}\right|}{\left|F_{n}\right|}=\sum_{x \in G / N} \frac{\left|F_{n} x_{n} \cap x N\right|}{\left|F_{n}\right|} \geq \sum_{x \in K} \frac{\left|F_{n} x_{n} \cap x N\right|}{\left|F_{n}\right|} \geq \frac{\delta}{2} \cdot|K| .
$$

Since $N$ has infinite index in $G$, we can choose $K \subset G / N$ so that the right hand side is strictly greater than one (for some index $n$ ), which is a contradiction.

We shall see that the case when the unitary representation is induced from $A$ is slightly trickier, and the argument is quite different than the one outlined in the last proof. To simplify matters, we shall impose an unnecessarily strong assumption on the subgroup $A$.

Proposition 7.2. Let $G$ be a countable group which splits as a semi-direct product of two subgroups $N$ and $A$ of $G$ with $N$ normal in $G$. If the action of $A$ on $N \backslash\{e\}$ has trivial stabilizers, then $A$ is thin with respect to any sequence of the form $\left(B_{n} A_{n}\right)$, where $\left(B_{n}\right)$ and $\left(A_{n}\right)$ are sequences of finite subsets of $N$ and $A$ respectively whose sizes both tend to infinity.

In particular, any such sequence is a firm strong sequence for any unitary representation of $G$ which is induced from a unitary representation of $A$.

Remark 7.1. The proposition above applies to the (countable) group $G_{K}$ of all affine transformations of a countable field $K$, which splits as a semidirect product of $K$ (translations) and $K^{*}$ (the multiplicative group of $K$ which acts by multiplication on $K$ ). The action of $K^{*}$ on $K \backslash\{0\}$ clearly has trivial stabilizers.

When $K=\mathbb{Q}$ (or more generally a finite extension thereof), J. Bost and A. Connes in their classical paper [6] constructed a family of irreducible unitary representations of $G_{K}$ which are not induced from subgroups of either $K$ or $K^{*}$. We do not know if any of these representations constitutes a counter-example to the Left Strong Ergodic Theorem for $G_{K}$, and we refer to the more recent paper [7] by T. Crisp for explicit formulas for these representations.

Proof. We note that since $G$ splits as a semidirect product of $N$ and $A$, it suffices to take the suprema in (7.1) over $x, y \in N$. Thus we want to prove that if the action of $A$ on $N \backslash\{e\}$ has trivial 
stabilizers, then for every choice of sequences $\left(s_{n}\right)$ and $\left(t_{n}\right)$ in $N$, we have

$$
\varlimsup_{n} \frac{\left|B_{n} A_{n} \cap s_{n} A t_{n}\right|}{\left|B_{n}\right| \cdot\left|A_{n}\right|}=\varlimsup_{n} \frac{\left|s_{n}^{-1} B_{n} A_{n} t_{n}^{-1} \cap A\right|}{\left|B_{n}\right| \cdot\left|A_{n}\right|}=0,
$$

whenever $\left(B_{n}\right)$ and $\left(A_{n}\right)$ are sequences of finite subsets of $N$ and $A$ respectively whose sizes tend to infinity. In other words, we wish to estimate the cardinality of the set of pairs $(b, a)$ in $B_{n} A_{n}$ which solves the equation

$$
s_{n}^{-1} b a t_{n}^{-1}=\left(s_{n}^{-1} b a t_{n}^{-1} a^{-1}\right) a \in A .
$$

or equivalently, $a t_{n} a^{-1}=s_{n}^{-1} b$, since $A \cap N=\{e\}$.

We shall separate between two cases. First, if $t_{n}=e$, then this set of solutions coincides with $s_{n} A_{n}$ and thus

$$
\frac{\left|B_{n} A_{n} \cap s_{n} A t_{n}\right|}{\left|B_{n}\right| \cdot\left|A_{n}\right|}=\frac{\left|s_{n} A_{n}\right|}{\left|A_{n}\right| \cdot\left|B_{n}\right|}=\frac{1}{\left|B_{n}\right|} .
$$

Since we assume that $\left|B_{n}\right|$ tends to infinity, we may without loss of generality assume that $t_{n} \neq e$ for all $n$.

In the second case, we assume that $t_{n} \neq e$ for all $n$. Since the action of $A$ on $N \backslash\{e\}$ has trivial stabilizers, there can be at most one solution to the equation $a t_{n} a^{-1}=s_{n}^{-1} b$ for every $s_{n}$ and $b$. Thus,

which finishes the proof.

$$
\frac{\left|B_{n} A_{n} \cap s_{n} A t_{n}\right|}{\left|B_{n}\right| \cdot\left|A_{n}\right|} \leq \frac{\left|s_{n}^{-1} B_{n}\right|}{\left|A_{n}\right| \cdot\left|B_{n}\right|}=\frac{1}{\left|A_{n}\right|} \rightarrow 0,
$$

\section{EMBEDdings INTO FLABBY PAIRS}

The aim of this section is to prove Theorem 1.4. We begin by recalling the notion of weak containment of unitary representations and some of its basic properties.

Definition 8.1 (Weak containment). Let $L$ be a countable group and let $\left(\mathscr{H}_{\pi}, \pi\right)$ and $\left(\mathscr{H}_{\rho}, \rho\right)$ be separable unitary representations of $L$. We say that $\pi$ is weakly contained in $\rho$, and write $\pi \prec \rho$, if for every vector $v \in \mathscr{H}_{\pi}$ and for every finite subset $F \subset L$ and $\varepsilon>0$, there exist vectors $w_{1}, \ldots, w_{k} \in \mathscr{H}_{\rho}$ such that

for all $s \in F$.

$$
\left|\langle v, \pi(s) v\rangle-\sum_{i=1}^{k}\left\langle w_{i}, \rho(s) w_{i}\right\rangle\right|<\varepsilon
$$

In particular, if $\pi$ denotes the identity representation on $\mathscr{H}_{\pi}=\mathbb{C}$, and $\pi$ is weakly contained in $\rho$, then there exists, for every finite subset $F \subset L$ and $\varepsilon>0$, vectors $w_{1}, \ldots, w_{k} \in \mathscr{H}_{\rho}$ such that

$$
\left|1-\sum_{i=1}^{k}\left\langle w_{i}, \rho(s) w_{i}\right\rangle\right|<\varepsilon, \quad \text { for all } s \in F .
$$

We stress that this is also the same as saying that the identity representation on any separable Hilbert space, e.g. $\ell^{2}(\mathbb{N})$, is weakly contained in $\rho$. We note that if $L$ is a countable amenable group and $\rho$ denotes the left regular representation on $\ell^{2}(L)$ and $\left(F_{n}\right)$ is any left Følner sequence in $L$, then the sequence

$$
w_{n}=\left|F_{n}\right|^{-1 / 2} \cdot \chi_{F_{n}}
$$

in $\ell^{2}(L)$ can be used to show that the identity representation is weakly contained in $\rho$. Furthermore, if $L$ is infinite, then the Hilbert space $\ell^{2}(L)$ admits no non-zero $\rho(L)$-invariant vectors. 
On the opposite side of amenability, we have the groups with Kazhdan's property (T), which we define here as follows.

Definition 8.2 (Kazhdan's Property (T)). A countable group $L$ has (Kazhdan's) property $(T)$ if whenever $(\mathscr{H}, \rho)$ is a unitary representation of $L$ which weakly contains the identity representation, then the Hilbert space $\mathscr{H}$ admits a non-zero $\rho(L)$-invariant vector.

Prominent examples of countable groups with property (T) are lattices in non-compact and connected simple Lie groups with finite center real rank at least two, e.g. $\mathrm{SL}_{n}(\mathbb{Z})$ for $n \geq 3$. For more details about these groups, we refer the reader to the book [5].

In order to prove Theorem [1.4, we shall make use of Proposition H.2 in the appendix of [14], which asserts that if $L$ is any countable group and if $\left(\mathscr{H}_{\pi}, \pi\right)$ and $\left(\mathscr{H}_{\rho_{o}}, \rho_{o}\right)$ are two infinite dimensional unitary representations of $L$ with $\pi<\rho_{o}$, then for every unit vector $v \in \mathscr{H}_{\pi}$, there exists a sequence $\left(\sigma_{n}\right)$ of unitary operators on the Hilbert space $\mathscr{H}_{\rho}=\oplus_{\mathbb{N}} \mathscr{H}_{\rho_{o}}$ and a unit vector $w \in \mathscr{H}_{\rho}$, such that

$$
\lim _{n}\left\langle w, \sigma_{n}^{-1} \rho(s) \sigma_{n} w\right\rangle=\langle v, \pi(s) v\rangle, \quad \forall s \in L,
$$

where $\rho=\rho_{o}^{\oplus \mathbb{N}}$ is the direct sum unitary representation on $\mathscr{H}_{\rho}$. We note that if $\rho_{o}$ does not admit non-zero invariant vectors, then neither does $\rho$.

In particular, if we apply all of this to the special case when $\pi$ is the identity representation on $\ell^{2}(\mathbb{N})$ and $\pi$ is weakly contained in $\rho_{o}$, then we have proved the following proposition.

Proposition 8.1 (Unitary orbits and weak containment). Let $L$ be a countable group without property $(T)$ and suppose that $\left(\mathscr{H}_{o}, \rho_{o}\right)$ is a unitary representation of $L$, which weakly contains the identity representation. Then there exists a (possibly different) unitary representation $(\mathcal{H}, \rho)$ of $L$, a unit vector $v \in \mathscr{H}$ and a sequence $\left(\sigma_{n}\right)$ of unitary operators on $\mathscr{H}$ such that

$$
\lim _{n}\left\langle v, \sigma_{n}^{-1} \rho(s) \sigma_{n} v\right\rangle=1,
$$

for all $s \in L$. Furthermore, if $\rho_{o}$ does not have invariant vectors, then neither does $\rho$.

Proof of Theorem 1.4 We recall that we want to prove that whenever $L$ does not have Kazhdan's property (T), then $L$ embeds into a countable group $G$ which admits a unitary representation $(\mathscr{H}, \tilde{\rho})$ with no non-zero $\tilde{\rho}(L)$-invariant vector, with the property that for some unit vector $v \in \mathscr{H}$ and for every sequence $\left(F_{n}\right)$ of finite subsets of $L$, there is a sequence $\left(s_{n}\right)$ in $G$ such that

$$
\lim _{n}\left\|\frac{1}{\left|F_{n}\right|} \sum_{s \in F_{n}} \tilde{\rho}\left(s s_{n}\right) v\right\|=1 .
$$

To prove this, we use the assumption that $L$ does not have property (T) to pick a unitary representation $\left(\mathscr{H}_{o}, \pi_{o}\right)$ of $L$ which weakly contains the identity representation, but which does not have any non-zero invariant vectors. Let $(\mathcal{H}, \rho)$ be the unitary representation of $L$ associated to $\left(\mathscr{H}_{o}, \pi_{o}\right)$ via Proposition 8.1 and let $\left(\sigma_{n}\right)$ be the corresponding sequence of unitary operators on $\mathscr{H}_{0}$. We denote by $S$ the countable subgroup of the group of unitary operators on $\mathscr{H}$ generated by the elements $\sigma_{n}$.

We let $G$ denote the free product of $L$ and $S$ and we note that by the universal property of free products, there exists a unitary representation $\tilde{\rho}$ of $G$ on the Hilbert space $\mathscr{H}$ which extends $\rho$ and satisfies $\tilde{\rho}(s)=s$ for all $s \in S$. Since $\rho$ does not have invariant vectors and $\tilde{\rho}$ extends the action of $\rho$ on the same Hilbert space, we conclude that $\tilde{\rho}$ does not admit any non-zero $L$-invariant vectors. 
We now fix a sequence $\left(F_{n}\right)$ of finite subsets of $L$ and we note that (8.1) guarantees that for every integer $n$, we can find an index $k_{n}$ such that

$$
\Re\left\langle v, \sigma_{k_{n}}^{-1} \rho(s) \sigma_{k_{n}} v\right\rangle \geq 1-\frac{1}{n} \text { for all } s \in F_{n}^{-1} F_{n},
$$

where $\Re$ denotes the real part of a complex number. In particular, we have

$$
\left\|\frac{1}{\left|F_{n}\right|} \sum_{s \in F_{n}} \tilde{\rho}\left(s \sigma_{k_{n}}\right) v\right\|^{2}=\frac{1}{\left|F_{n}\right|^{2}} \sum_{(s, t) \in F_{n} \times F_{n}} \Re\left\langle v, \sigma_{k_{n}}^{-1} \rho\left(s^{-1} t\right) \sigma_{k_{n}} v\right\rangle \geq 1-\frac{1}{n},
$$

which finishes the proof.

\section{EXAMPLES OF CONTRACTING TRIPLES AND CONJUGATION-THICK SUBGROUPS}

In this section we shall give two classes of examples of (left amenable) contracting triples and thereby supplying explicit examples of triples (and groups) for which the Left Strong Ergodic Theorem fails in the strongest possible way.

9.1. HNN-extensions. The first class of examples stems from special cases of HNN-extensions. Suppose that we are given a countable group $H$ and an injective homomorphism $\alpha: H \rightarrow H$. We define the countable group

$$
\left.G_{\alpha}=\langle H, t| t h t^{-1}=\alpha(h) \quad \text { for all } h \in H\right\rangle,
$$

that is to say, $G_{\alpha}$ is the group generated by $H$ and the cyclic group generated by the element $t$, which is assumed to satisfy the relation $t h t^{-1}=\alpha(h)$ for all $h$ in $H$. We note that once $G_{\alpha}$ has been defined, the subgroups $H_{k}=t^{-k} H t^{k}$ form an ascending chain of subgroups of $G_{\alpha}$, i.e.

$$
\ldots<H_{-2}<H_{-1}<H<H_{1}<H_{2}<\ldots
$$

If we set $L_{\alpha}=\cup_{k} H_{k}$, then $G_{\alpha}$ splits as a semidirect product of the normal subgroup $L_{\alpha}$ and the cyclic subgroup generated by $t$. In particular, if $H$ is amenable, then so is $G_{\alpha}$.

For instance, we could start with the group $H=\mathbb{Z}$ with the injective homomorphism $\alpha(n)=$ $p n$, where $p$ is some non-zero integer. In this case, the group $L_{\alpha}$ coincides with $Z[1 / p]$ and $G_{\alpha}$ is isomorphic to the (outer) semi-direct product $Z[1 / p] \rtimes \mathbb{Z}$, where $\mathbb{Z}$ acts on $Z[1 / p]$ by multiplication with powers of $p$.

We shall now prove the following simple proposition about these groups.

Proposition 9.1. For every countable group $H$ and injective homomorphism $\alpha: H \rightarrow H$, the triple $\left(G_{\alpha}, H, L_{\alpha}\right)$ is contracting. Furthermore, if $\alpha(H) \neq H$, then $H$ has infinite index in $L_{\alpha}$.

Proof. We fix a finite subset $F \subset L_{\alpha}$. By the ascending chain (9.1) of subgroups, there exists an index $k$ such that $F \subset H_{k}=t^{-k} H t^{k}$, and thus $t^{k} F t^{-k} \subset H$, which shows that $\left(G_{\alpha}, H, L_{\alpha}\right)$ is contracting. The second part follows from the multiplicativity of the index; note that for every positive integer $k$, we have

$$
\left[H_{k}: H\right]=\left[H_{k}: H_{k-1}\right] \cdot\left[H_{k-1}: H\right]=\ldots=\prod_{j=0}^{k-1}\left[H_{j+1}: H_{j}\right],
$$

and

$$
\left[H_{j+1}: H_{j}\right]=\left[t^{-(j+1)} H t^{(j+1)}: t^{-j} H t^{j}\right]=\left[t^{-1} H t: H\right]=\left[H_{1}: H\right],
$$

for every $j$, where the second equality is justified by the fact that conjugation by $t^{-j}$ does not change the index. In particular, we see that $H$ has infinite index in $L$ whenever $H$ is a proper subgroup of $H_{1}$, which is the case if $\alpha(H) \neq H$. 
9.2. Permutations with finite supports. We shall now show that the countable group $\operatorname{Sym}_{o}(\mathbb{N})$ consisting of those permutations $s$ on $\mathbb{N}$ for which the support

$$
\operatorname{supp}(s)=\{x \in \mathbb{N}: s(x) \neq x\}
$$

is finite, admits conjugation-thick subgroups. We note that $\operatorname{Sym}_{o}(\mathbb{N})$ can be written as a union of an increasing chain of finite subgroups, that is to say, it is locally finite and thus amenable.

Proposition 9.2. For every non-empty finite set $K \subset \mathbb{N}$, the subgroup

$$
L_{K}=\left\{s \in \operatorname{Sym}_{o}(\mathbb{N}):\left.s\right|_{K}=i d\right\}<\operatorname{Sym}_{o}(\mathbb{N}) \text {. }
$$

is a proper conjugation-thick subgroup of $\operatorname{Sym}_{o}(\mathbb{N})$.

Proof. We fix a finite set $K \subset \mathbb{N}$ and pick a finite subset $F \subset \operatorname{Sym}_{o}(\mathbb{N})$. We want to show that there exists an element $\sigma \in \operatorname{Sym}_{o}(\mathbb{N})$ such that $\sigma F \sigma^{-1} \subset L_{K}$, or equivalently $F \subset L_{\sigma^{-1}(K)}$. To prove this, we pick any finite subset

$$
M \subset \mathbb{N} \backslash \bigcup_{s \in F} \operatorname{supp}(s)
$$

with $|M|=|K|$ and choose an involution $\sigma$ in $\operatorname{Sym}_{o}(\mathbb{N})$ which maps $M$ onto $K$ and leaves all elements outside $M \cup K$ untouched. Clearly, $F \subset L_{M}=L_{\sigma^{-1}(K)}$, which finishes the proof.

\section{Automatic POINTWISE ERGODIC THEOREMS}

We begin by describing two classes of rigid pairs of countable groups.

Example (Affine groups over countable fields). Let $\mathbb{K}$ be a countable (infinite) field and let $G_{\mathbb{K}}$ denote the semidirect product of the additive group $\mathbb{K}$ and the multiplicative group $\mathbb{K}^{*}$, where the latter acts on $\mathbb{K}$ by multiplication. Suppose $(\mathscr{H}, \pi)$ is a unitary representation of $G_{\mathbb{K}}$ without any $\pi\left(G_{\mathbb{K}}\right)$-invariant vectors, but with a $\pi\left(\mathbb{K}^{*}\right)$-invariant unit vector $v_{o}$. We define

$$
\phi(b, a)=\left\langle\pi(b, a) v_{o}, v_{o}\right\rangle, \quad \text { for } b \in \mathbb{K} \text { and } a \in \mathbb{K}^{*},
$$

and note that since $v_{o}$ is $\mathbb{K}^{*}$-invariant, the function $\phi$ is independent of the second coordinate and satisfies

$$
\phi(a \cdot b, 1)=\phi(b, 1) \quad \text { for all } b \in \mathbb{K} \text { and } a \in \mathbb{K}^{*} .
$$

Since every non-zero $\mathbb{K}^{*}$-orbit in $\mathbb{K}$ equals $\mathbb{K}$, we conclude that

$$
\phi(b, a)=\left\{\begin{array}{cc}
1 & \text { if } b=0 \\
\phi(1,1) & \text { otherwise }
\end{array} .\right.
$$

It is now a straightforward exercise to verify that $\phi(1,1)=0$ using the Left Weak Ergodic Theorem and the assumption that $(\mathscr{H}, \pi)$ does not admit any non-zero $\pi(G)$-invariant vectors. We conclude that the pair $\left(G_{\mathbb{K}}, \mathbb{K}^{*}\right)$ is rigid.

Example (Character rigid groups). Let $G_{o}$ be a countable group and define the pair

$$
G=G_{o} \times G_{o} \text { and } H=\Delta_{2}\left(G_{o}\right)
$$

where the latter group denotes the diagonal subgroup in $G$. If $(\mathscr{H}, \pi)$ is a unitary representation of $G$ with a $\pi(H)$-invariant unit vector $v_{o}$, then one can readily verify that

$$
\phi(s, t)=\left\langle v_{o}, \pi(s, t) v_{o}\right\rangle \quad \text { for }(s, t) \in G
$$

satisfies $\phi(s, t)=\phi_{o}\left(s^{-1} t\right)$ for all $s, t \in G_{o}$, where $\phi_{o}(t)=\phi(e, t)$, and $\phi_{o}$ is a conjugation-invariant function on $G_{o}$ with $\phi_{o}(e)=1$. Functions of this form are often referred to as characters in $G_{o}$, and we say that $G_{o}$ is character rigid if every character which is not constant is simply the indicator function of the identity element. Clearly, the pair $(G, H)$ is rigid if and only if $G_{o}$ is character rigid. 
Recently it has been proved by A. Thom and J. Peterson in [21] that the groups $\mathrm{SL}_{n}(R)$ are character rigid for $n \geq 2$ and for a wide variety of infinite rings $R$, including all infinite fields, and A. Dudko and K. Medynets have proved that the Higman-Thompson group is character rigid.

We shall now give the short proof of Theorem 1.6. Let $(G, H)$ is a rigid pair and suppose that $(X, v)$ is an ergodic probability measure preserving $G$-space. We note that $G$ acts in a unitary way on $L_{o}^{2}(X, v)$ (equivalence classes of square-integrable functions with zero integral) via the left regular representation (Koopman representation). If $\varphi \in L_{o}^{2}(X, v)$ is $H$-invariant, then

$$
\int_{X} \varphi(x) \overline{\varphi\left(s^{-1} \cdot x\right)} d v(x)=0
$$

for all $s \notin H$ by the assumption that $(G, H)$ is rigid.

Let $\left(F_{n}\right)$ be any strictly increasing and nested sequence of finite subsets of $G / H$ so that we can write

$$
F_{n}=\left\{s_{1} H, \ldots, s_{m_{n}} H\right\}
$$

for some sequence $m_{n}$ of integers which tend to infinity, and distinct elements $s_{k} H$ in $G / H$. We set

$$
\varphi_{k}(x)=\varphi\left(s_{k}^{-1} x\right) \quad \text { for } k \geq 1
$$

and note $\left(\varphi_{k}\right)$ is a sequence of orthogonal vectors in $L^{2}(X, v)$ with

$$
\int_{X}\left|\varphi_{k}\right|^{2} d v=\int_{X}|\varphi|^{2} d v \text { for all } k
$$

Theorem 1.6 is now an immediate consequence of the following rather standard lemma in measure theory (sometimes attributed to A. Rajchman) whose proof we include here for completeness.

Lemma 10.1. Let $(X, v)$ be a probability measure space and suppose that $\left(\varphi_{k}\right)$ is sequence of orthonormal vectors in $L_{o}^{2}(X, v)$. There exists a conull subset $X^{\prime} \subset X$ such that

$$
\lim _{n} \frac{1}{n} \sum_{k=1}^{n} \varphi_{k}(x)=0
$$

for all $x \in X^{\prime}$.

Proof. Since

$$
\sum_{n=1}^{\infty}\left\|\frac{1}{n^{2}} \sum_{k=1}^{n^{2}} \varphi_{k}\right\|_{2}^{2}=\sum_{n=1}^{\infty} \frac{1}{n^{2}}<\infty
$$

we conclude by Borel-Cantelli's Lemma, that

$$
\lim _{n} \frac{1}{n^{2}} \sum_{k=1}^{n^{2}} \varphi_{k}(x)=0
$$

for all $x$ in some $v$-conull subset $X_{1} \subset X$.

Given an integer $m$, we denote by $n(m)$ the unique positive integer with

$$
n(m)^{2} \leq m \leq(n(m)+1)^{2},
$$

so that

$$
\frac{1}{m^{2}} \int_{X}\left|\sum_{k=1}^{m} \varphi_{k}-\sum_{k=1}^{n(m)^{2}} \varphi_{k}\right|^{2} d v \leq \sum_{m=1}^{\infty} \frac{m-n(m)^{2}}{m^{2}} \leq \sum_{m=1}^{\infty}\left(m^{-2}+2 \cdot m^{-\frac{3}{2}}\right)<\infty
$$


for all $m$, since

$$
m-n(m)^{2} \leq(n(m)+1)^{2}-n(m)^{2}=1+2 n(m) \leq 1+2 \sqrt{m} .
$$

This shows, again by Borel-Cantelli' Lemma, that

$$
\lim _{m} \frac{1}{m}\left(\sum_{k=1}^{m} \varphi_{k}(x)-\sum_{k=1}^{n(m)^{2}} \varphi_{k}(x)\right)=0
$$

for all $x$ in some $v$-conull subset $X_{2} \subset X$. Hence,

$$
\lim _{m} \frac{1}{m} \sum_{k=1}^{m} \varphi_{k}=\lim _{m} \frac{1}{m} \cdot\left(\sum_{k=1}^{m} \varphi_{k}(x)-\sum_{k=1}^{n(m)^{2}} \varphi_{k}(x)\right)+\lim _{m} \frac{n(m)^{2}}{m} \cdot\left(\frac{1}{n(m)^{2}} \sum_{k=1}^{n(m)^{2}} \varphi_{k}(x)\right)=0,
$$

for all $x \in X^{\prime}=X_{1} \cap X_{2}$, where the last equality follows from the fact that the ratio between the numbers $n(m)^{2}$ and $m$ tends to one.

\section{APPENDIX: FiRM STRONG SEQUENCES IN LATTICES IN HIGHER RANK LIE GROUPS}

We shall now derive the formulation of Proposition 1.2 from that of Theorem 1.7 in [13].

Let $G$ be a lattice in a non-compact and connected simple Lie group $\tilde{G}$ with trivial center. Theorem 1.7 in [13] asserts there exists a sequence $\left(F_{n}\right)$ of finite subsets of $G$ (which can be chosen as the intersections of $G$ with an increasing family of geometrically defined balls in the ambient Lie group $\tilde{G}$ ) with the property that for every ergodic probability measure preserving $G$-space $(X, v)$, there exist constants $\delta>0$ and $C$ such that

$$
\sup \left\{\left\|\frac{1}{\left|F_{n}\right|} \sum_{s \in F_{n}} \pi(s) f\right\|_{2}:\|f\|_{2}=1\right\} \leq C \cdot e^{-\delta n}
$$

for all $n$, where $\pi$ denotes the left regular representation (Koopman representation) of $G$ on the Hilbert space $L_{o}^{2}(X, v)$. This clearly shows that $\left(F_{n}\right)$ is a firm strong sequence for left regular (Koopman) representations associated to any ergodic probability measure preserving $G$-space.

In particular, if $K$ is a compact group with Haar probability measure $m_{K}$ and $\pi: G \rightarrow K$ is a homomorphism with dense image in $K$, then the associated probability measure preserving $G$-action on $\left(K, m_{K}\right)$ given by $s \cdot k=k \pi\left(s^{-1}\right)$ is ergodic, and thus

$$
\lim _{n} \frac{1}{\left|F_{n}\right|} \sum_{s \in F_{n}} \int_{K} \psi(k) \phi\left(k \pi\left(s s_{n}\right)\right) d m_{K}(k)=\int_{K} \psi d m_{K} \cdot \int_{K} \phi d m_{K}
$$

for all real-valued continuous functions $\psi$ and $\phi$ on $K$ and for every sequence $\left(s_{n}\right)$ in $G$.

We shall now show that this simple observation implies that $\left(F_{n}\right)$ is a firm strong sequence for every finite-dimensional unitary representation of $G$. Indeed, suppose that $(\mathscr{H}, \pi)$ is such a representation and let $K$ denote the compact closure of $\pi(G)$ in the orthogonal group of $\mathscr{H}$. We may assume that $\mathscr{H}$ does not admit any non-zero $\pi(G)$-invariant vectors. Since the weak topology and the norm topology on $\mathscr{H}$ coincide, it suffices to show that

$$
\lim _{n} \frac{1}{\left|F_{n}\right|} \sum_{s \in F_{n}}\left\langle w, \pi\left(s s_{n}\right) v\right\rangle=0
$$

for all $v$ and $w$ in a weakly dense subspace of $\mathscr{H}$ and for every sequence $\left(s_{n}\right)$ in $G$.

We write $k \cdot v$ for the image of a vector $v$ under the action of an element $k$ in $K$, so in particular we have $\pi(s) v=\pi(s) \cdot v$ for all $s$ in $G$. We note that for every $v, w \in \mathscr{H}$, the bounded function $\phi$ on $G$ defined by

$$
\phi(s)=\langle w, \pi(s) v\rangle, \quad s \in G,
$$


can be extended to a continuous function $\tilde{\phi}$ on $K$, and the linear space of all vectors in $\mathscr{H}$ of the form

$$
w_{\psi}=\int_{K} \psi(k) k^{-1} \cdot w d m_{K}(k)
$$

as $\psi$ ranges over all continuous functions on $K$ and $w$ over all elements in $\mathscr{H}$ is weakly dense. By our observation in (10.2), we have

$$
\lim _{n} \frac{1}{\left|F_{n}\right|} \sum_{s \in F_{n}}\left\langle w_{\psi}, \pi\left(s s_{n}\right) v\right\rangle=\lim _{n} \frac{1}{\left|F_{n}\right|} \sum_{s \in F_{n}} \int_{K} \psi(k) \tilde{\phi}\left(k \pi\left(s s_{n}\right)\right) d m_{K}=\int_{K} \psi d m_{K} \cdot \int_{K} \tilde{\phi} d m_{K},
$$

so it suffices to show that the right hand side vanishes. However, one readily verifies that the integral of $\tilde{\phi}$ equals $\left\langle w, P_{G} v\right\rangle$, where $P_{G}$ denotes the projection onto the $\pi(G)$-invariant vectors in $\mathscr{H}$, which by assumption is trivial, and thus the right hand side vanishes.

If $(\mathscr{H}, \pi)$ is a weakly mixing unitary representation of $G$, then we can use the Gaussian measure construction (see e.g. Appendix $\mathrm{C}$ in [14]) to realize every cyclic sub-representation of $(\mathscr{H}, \pi)$ as a sub-representation of the Koopman representation associated to an ergodic (for this weak mixing is crucial) probability measure preserving $G$-space, and Proposition 1.2 follows.

\section{REFERENCES}

1. C. Anatharaman-Delaroche, Approximation properties of coset spaces and their operator algebras. Preprint.

2. L. Bartholdi, R.I. Grigorchuk, Z. Sunik. Branch groups. Preprint.

3. M. Beiglböck, V. Bergelson, A. Fish, Sumset phenomenon in countable amenable groups, Adv. Math. 223 No. 2 (2010), 416-432.

4. M. Bekka, M, P. de la Harpe, Représentations d'un groupe faiblement équivalentes á la représentation réguliére. (French. English, French summary) [Representations of a group that are weakly equivalent to the regular representation] Bull. Soc. Math. France 122 (1994), no. 3, 333-342.

5. B. Bekka, P. de la Harpe, A. Valette, Kazhdan's property (T). New Mathematical Monographs, 11. Cambridge University Press, Cambridge, 2008. xiv+472 pp. ISBN: 978-0-521-88720-5

6. J.B. Bost, A. Connes, Hecke algebras, type III factors and phase transitions with spontaneous symmetry breaking in number theory, Selecta Math. (N.S.) 1 (1995), 411-457.

7. T. Crisp, The Bost-Connes phase transition and unitary representations. Journal of Noncommutative Geometry 7 no. 1 (2013) 291-300.

8. E.K. van Douwen, Measures invariant under actions of $F_{2}$, Topology Appl. 34 (1990), no. 1, 53-68.

9. A. Dudko, K. Medynets, Finite factor representations of Higman-Thompson groups. Groups Geom. Dyn. 8 (2014), no. 2, 375-389.

10. W.F.Eberlein, Abstract ergodic theorems and weak almost periodic functions, Trans. Am. Math. Soc, 67 (1949), 217-240.

11. W. Emerson, F. Greenleaf, Group structure and the pointwise ergodic theorem for connected amenable group. Adv. Math. 14 (1974), 153-172.

12. Y. Glasner, N. Monod, Amenable actions, free products and a fixed point property, Bull. London Math. Soc. 39 No. 1 (2007), 138-150.

13. A. Gorodnik and A. Nevo, The ergodic theory of lattice subgroups, Annals of Mathematics Studies 172, Princeton University Press, 2010.

14. A. Kechris, Global aspects of ergodic group actions. Mathematical Surveys and Monographs, 160. American Mathematical Society, Providence, RI, 2010. xii+237 pp. ISBN: 978-0-8218-4894-4.

15. A. Kechris, B. Miller, Topics in orbit equivalence. Lecture Notes in Mathematics, 1852. Springer-Verlag, Berlin, 2004. $\mathrm{x}+134$ pp. ISBN: 3-540-22603-6

16. A. Lubotsky, Discrete groups, expanding graphs and invariant measures, Progress in Math., Birkhauser, 1994

17. G. W. Mackey, On induced representations of groups, Amer. J. Math. 73 (1951), 576âĂŞ592.

18. N. Monod, S. Popa, On co-amenability for groups and von Neumann algebras, C. R. Acad. Sci. Canada 25 No. 3 (2003), 82-87

19. I. Namioka, Følner's conditions for amenable semi-groups, Math. Scand. 15 (1964), 18-28.

20. A. Nevo, Harmonic analysis and pointwise ergodic theorems for noncommuting transformations. J. Amer. Math. Soc. 7 (1994), no. 4, 875-902. 
21. J. Peterson, A. Thom, Character rigidity for special linear groups, Accepted to J. Reine Angew. Math. (Crelle's Journal).

22. F. Riesz, Sur la théorie ergodique. Comm. Math. Helv. 17 (1945), 221-239.

23. W. Rudin, Fourier analysis on groups. Reprint of the 1962 original. Wiley Classics Library. A Wiley-Interscience Publication. John Wiley \& Sons, Inc., New York, 1990. x+285 pp. ISBN: 0-471-52364-X

24. K. Schmidt, Asymptotic properties of unitary representations and mixing. Proc. London Math. Soc. (3) 48 (1984), no. $3,445-460$.

25. E. Thoma, Über unitäre Darstellungen abzählbarer diskreter Gruppen, Math. Ann. 153 (1964), 111-138.

26. A. Vershik, Nonfree actions of countable groups and their characters. J. Math. Sci. (N. Y.) 174 (2011), no. 1, 1-6

27. J. von Neumann, Proof of the quasiergodic hypothesis. Proc. Nat. Acad. Sci. (U.S.A.) 18 (1932), 70-82.

28. R. Zimmer, Ergodic theory and semisimple groups. Monographs in Mathematics, 81. Birkhäuser Verlag, Basel, 1984. $\mathrm{x}+209$ pp. ISBN: 3-7643-3184-4

Department of Mathematics, Chalmers, Gothenburg, Sweden

E-mail address: micbjo@chalmers.se

School of Mathematics and Statistics, University of Sydney, Australia

E-mail address: alexander.fish@sydney.edu.au 